\title{
Flow Around a Cube in a Turbulent Boundary Layer: LES and Experiment
}

\author{
Hee Chang Lim, ${ }^{*}$ T. G. Thomas \& Ian P Castro \\ School of Engineering Sciences, University of Southampton, UK
}

\begin{abstract}
We present a numerical simulation of flow around a surface mounted cube placed in a turbulent boundary layer which, although representing a typical wind environment, has been specifically tailored to match a series of wind tunnel observations. The simulations were carried out at a Reynolds number, based on the velocity $U$ at the cube height $h$, of 20,000 - large enough that many aspects of the flow are effectively Reynolds number independent. The turbulence intensity was about $18 \%$ at the cube height, and the integral length scale $L_{x}^{u}$ was about 0.8 times the cube height $h$. The Jenson number $J e=h / z_{0}$, based on the approach flow roughness length $z_{0}$, was 600 , to match the wind tunnel situation. The computational mesh was uniform with a spacing of $h / 32$, aiding rapid convergence of the multigrid solver, and the governing equations were discretised using second order finite differences within a parallel multiblock environment. The results presented include detailed comparison between measurements and LES computations of both the inflow boundary layer and the flow field around the cube including mean and fluctuating surface pressures. It is concluded that provided properly formulated inflow and surface boundary conditions are used, LES is now a viable tool for use in wind engineering problems concerning flow over isolated bodies. In particular, both mean and fluctuating surface pressures can be obtained with a similar degree of uncertainty as usually associated with wind tunnel modelling.
\end{abstract}

Key words: Bluff body, Large eddy simulation, Wind environment

\section{Introduction}

The use of various forms of Computational Fluid Dynamics (CFD) in the general field of Wind Engineering has become widespread over the last decade or so but it is generally recognized that the standard turbulence models used in most codes (e.g. eddy

* Present address: School of Mechanical Engineering, PuKyong National University, South Korea

Preprint submitted to Elsevier

January 2008 
viscosity-based models like $k-\epsilon$, or the various second-order stress models) are seriously inadequate in many respects, particularly for studying flow around bluff obstacles like buildings, bridges, etc (see Castro \& Graham, 1999). Unsteady techniques like Discrete Vortex Methods and, particularly, Large Eddy Simulation (LES), are in principle much more appropriate and are in any case imperative for proper identification of the important unsteady features of such flows. As long as two decades ago Murakami et al. (1987) showed the potential for LES computations for the case of a boundary layer flow over a cube, although by today's standards their grid was extremely coarse and there was undoubtedly considerable numerical dissipation. Shah \& Ferziger $(1996,1997)$ completed a more rigorous computation of a similar flow and demonstrated that LES 'is capable of providing information of importance to wind engineers about flows over bluff bodies'. In his more recent review of LES for bluff-body flows, which compared LES and RANS predictions of a cube-in-a-channel flow, Rodi (2002) demonstrated that 'on the whole, LES is able to simulate this complex flow very well'. This was in contrast to the situation for flow past a two-dimensional square cylinder, which turns out (still) to be a significantly more taxing problem. It is worth noting that Rodi's own computations of the cube flow used a grid spacing near the cube of around $0.0125 \mathrm{~h}$ - an order of magnitude smaller than in Murakami's (1987) computations. It is now generally accepted that such spacings cannot be greatly exceeded without serious accuracy implications. Overall, the LES captured all the complex features of the cube flow quite well, even quantitatively. However, these more recent simulations have invariably been for a cube in a smooth-walled channel, using precursor computations of channel flow to provide the time-dependent inlet conditions. They have also concentrated largely on the mean flow, with relatively little attention paid to how well the unsteady features of the flow are predicted.

The structurally significant (local) loads exerted by turbulent flow on typical bluff bodies are mainly due to the extreme low pressure regions created within the flow by the shedding of concentrated vorticity. Peak (instantaneous) pressures are often reckoned to be the result of particular upstream gusts leading to strong but short-term vortex generation at the body's leading edges. LES is ideally suited to such problems as it aims to compute directly the dynamically significant flow structures. Attempts have recently begun to compute these unsteady features. Nozawa \& Tamura (2002), for example, have used LES to determine mean and fluctuating surface pressures on a half-cube model. Although they used a precursor simulation to generate appropriate rough-wall boundary layer inflow conditions, the ground surface in the computational domain used for the flow over the model was actually smooth. Nonetheless, they found reasonable agreement with experimental data, also obtained over a smooth surface. Ono et al. (2006) have done an LES analysis of conical vortex structures generated on the roof of a flat building at $45^{\circ}$ to the approach flow. However, there are many features of LES which, in its application to environmental bluff body flows, remain contentious or, at best, unresolved. These include the issue of how to apply appropriate rough-wall boundary conditions, the way in which inflow conditions should be defined (particularly taxing in the Wind Engineering context, when the upstream flow is itself fully turbulent and sheared) and the importance or otherwise of the sub-grid scale formulation. It is reasonable to suppose, for example, that the unsteady pressure loading on the body comes partly from the distortion of vortex structures embedded in the inflow as they interact with the body and are deformed and stretched. This suggests that it is important to quantify the structure of the inflow turbulence in more detail than is usually done and to examine how identifiable structure 
in the upstream flow is related to 'loading events' on the body. Regarding the sub-grid modeling issue, this is presumably particularly crucial for those relatively small-scale regions of the flow where the extremely low (fluctuating) pressures are generated e.g. the leading edge vortices attached at upstream corners and the roll-up products of the thin shear layers which detach from the body. Krajnovic (1999) has shown, for the cube test case referred to earlier, that for fairly high Reynolds numbers (40,000 in his case) the influence of the subgrid-scale model can be important.

Our overall objective in the present study is to clarify the extent to which LES, on even relatively moderate grids, can capture the major features of the flow around a three-dimensional bluff body submerged in a thick, rough-wall, simulated atmospheric boundary layer. We simulate the flow around a surface mounted cubic obstacle placed in a turbulent boundary layer, with the aim that it represent a typical wind environment but be specifically designed to match the companion wind tunnel experiment (Lim et al. 2007) as closely as possible. We believe this is the first time such controlled and matched experiments and computations have been performed for a realistic (albeit relatively low Reynolds number) case of this kind. The emphasis is on the comparisons between the LES and wind tunnel data, so physical features of the (largely well-known) nature of the flow around the body are (mostly) discussed only insofar as they may explain any differences between the physical and numerical experiments.

The importance of modelling the details of the upstream flow in wind tunnel simulations is well known; by implication the same must be true in numerical simulations. Accordingly, we perform a separate (precursor) simulation of the approach flow and adopt a Schumann-type wall condition specifically adapted (Thomas \& Williams, 1999) for the rough surface used in the wind tunnel experiments. An alternative approach would be to use one of the many digital filter methods, in which inlet turbulence of the required intensity and spatial scale (in all coordinate directions) is produced by carefully massaging sets of random numbers (e.g. Klein et al. 2003). Note that in some reported wind engineering experiments, although the simulated atmospheric boundary layer included a surface covered with appropriate roughness elements, these were removed in the region around the building model (a cube, say), usually because they were not very small compared with the cube size. But this introduces issues concerning the change of surface condition and how that might affect the flow around the body. Similar problems would occur in numerical computations which attempted to do the same. In this work, the roughness size was small compared to the body size and roughness was thus retained all around the cube.

The calculations were carried out at a Reynolds number, $R e_{h}$, based on the velocity $U_{h}$ at the cube height $h$, of 20,000 - large enough that in some respects at least the flow is effectively Reynolds number independent (but see Lim et al. 2007). The Jenson number $J e=h / z_{0}$, based on the approach roughness length $z_{0}$, is 600 approximately matching the wind tunnel data. The experimental and numerical techniques are described in $\S 2.1$ and $\S 2.2$, respectively. Results are presented in $\S 3$ which includes first, in $\S 3.1$, discussion of the upstream boundary layer, followed by a brief overview of the overall flow ( $\$ 3.2)$ and then more detailed discussion of the flow around the cube (§3.3), where emphasis is placed on specific quantitative comparisons between computed and measured mean and fluctuating quantities. 


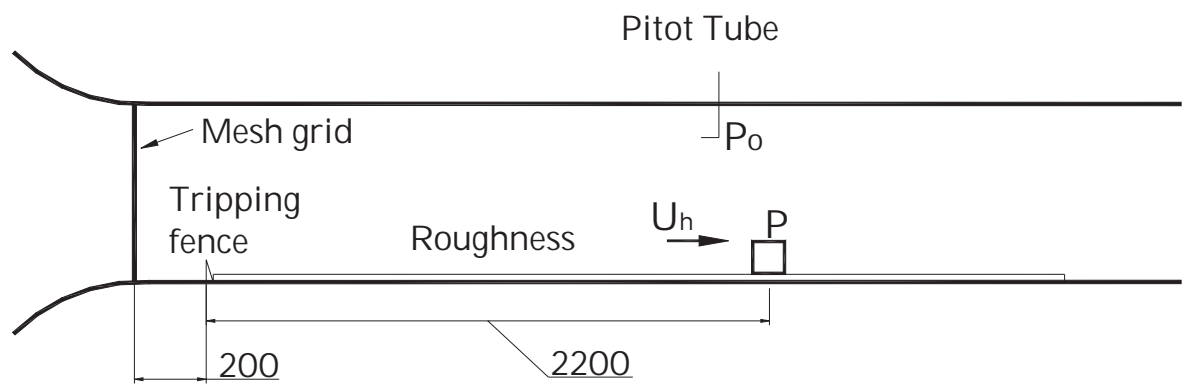

Fig. 1. The $3^{\prime} \times 2$ ' wind tunnel test section and setup. Dimensions in $\mathrm{mm}$.

\section{Techniques}

\subsection{Laboratory experiments}

Figure 1 illustrates the set-up, showing the cube location in the $3^{\prime} \times 2^{\prime}$ wind tunnel, which is an open circuit facility with a $0.9 \mathrm{~m} \times 0.6 \mathrm{~m} \times 4.5 \mathrm{~m}$ working section having a maximum wind speed of about $40 \mathrm{~m} \mathrm{~s}^{-1}$. It is suited for generating an artificial boundary layer and is also equipped with modern hot-wire anemometry, a 2-axis LDA system and a PIV system for optical measurements of the airflow. Full details of the experimental techniques are provided in Lim et al. (2007) and here we merely summarise the major points.

\subsubsection{The simulated atmospheric boundary layer}

The atmospheric boundary layer (abl) simulation was carried out using a combination of mesh roughness, barrier and mixing device (a grid), which is a conventional technique for generating such a flow (Cook, 1978). Toothed barriers spanning the floor of the working section near its entry, followed by a square section, bi-planar mesh across the entire working section and an appropriate rough surface thereafter, can together be designed to yield mean velocity profiles which are closely logarithmic over a significant portion of the working section height, with turbulence stresses and spectra similar to those found in atmospheric, neutrally stable boundary layers. It is crucial to design the barrier wall and mixing grid geometries in tandem with the intended roughness, since any mismatch will yield unacceptably long fetches before reasonably well-developed flows are attained. In the present case, commercially available expanded aluminium mesh was used to provide the surface roughness. This gave a roughness length, $z_{o}$, of $0.09 \mathrm{~mm}$, where $z_{o}$ is defined in the usual way via the mean velocity log law expressed as:

$$
\frac{U}{u_{*}}=\frac{1}{\kappa} \ln \frac{(z-d)}{z_{0}}
$$

where $u_{*}$ and $d$ are the friction velocity $\left(\sqrt{\tau_{\text {wall }} / \rho}\right)$ and 'zero plane displacement', respectively. Obtaining the three unknowns $\left(u_{*}, d\right.$ and $\left.z_{o}\right)$ from the mean velocity profile alone is, as is well-known, a very ill-conditioned process. In the present study, $u_{*}$ was deduced from an extrapolation of the measured turbulence shear stress $(-\overline{u w})$ to the surface (see Lim et al. 2007), with $d$ and $z_{0}$ then following from a best fit of the mean velocity data to eq.(1). 


\subsubsection{Surface pressure measurement}

A cube model of height $(h) 80 \mathrm{~mm}$, constructed from MDF (Medium Density Fibre) of $12 \mathrm{~mm}$ thickness, was fitted with $0.8 \mathrm{~mm}$ i.d. pressure taps at numerous salient points on the top surface and the front and rear faces. Standard tube connections to a (Furness, FC-012) micromanometer allowed measurement of mean surface pressures. Fluctuating pressures were obtained using both piezo-resistive sensors (Endevco, 8507C-2) and omni-directional condenser-type microphones (Panasonic WM-60A). The former had a diameter of $1.27 \mathrm{~mm}$ and a frequency response which was flat from dc to around $15 \mathrm{kHz}$, whereas the latter had a $6 \mathrm{~mm}$ diameter and so were each mounted in a small cavity beneath a $0.5 \mathrm{~mm}$ pinhole in the surface, yielding a response which was flat between 20 $\mathrm{Hz}$ and $20 \mathrm{kHz}$.

To measure mean surface pressure distributions, 23 static taps were installed flush with the surfaces of the cube model. At each measurement point, 50 blocks of 4,096 pressure samples were acquired at a $2 \mathrm{kHz}$ sampling rate after low-pass filtering at $1.6 \mathrm{kHz}$. A time delay of a few seconds was given to recover correct pressure fluctuations after scanning each channel. The difference between the surface pressure $p$ on the cube surface and the static pressure $p_{0}$ measured at the reference position by a Pitot-static probe upstream of the cube was non-dimensionalised using $U_{h}$, the velocity at the cube height (but in its absence) to give the pressure coeffcient, $C_{p}$, in the usual form:

$$
C_{p}=\frac{p-p_{0}}{\frac{1}{2} \rho U_{h}^{2}}
$$

\subsubsection{Velocity measurement}

Mean velocity and turbulence stress data within the boundary layer at the cube location and around the cube itself were obtained using appropriate combinations of hot wire (HWA), laser doppler (LDA) and particle image (PIV) anemometry systems. Hot wire probes (including crossed wires of $\pm 60^{\circ}$ to minimise high turbulence errors) were calibrated against a standard Pitot-static tube using the same micromanometer as used for the (static) pressure measurements. All analogue signals were digitised, passed to a (Macintosh) desk-top computer and analysed on-line using 'virtual instruments' written in National Instruments' LabVIEW). Probes were supported on traverse systems driven by the same computer. Sampling rates were typically between $2 \mathrm{kHz}$ and $10 \mathrm{kHz}$, depending on the quantities being measured, with sample times of 60-120 s. For LDA, a two-component, fibre-optic Dantec system was used, with Burst Spectrum Analysers (BSA) to process the Doppler bursts. Since turbulence intensities close to the surface and near the cube were often greatly in excess of $25 \%$, interval time weighting was used to minimise bias errors. The probe was located outside the working section, with the beams transmitted through the perspex side walls.

In order to provide data for comparison with the Large Eddy Simulations, PIV has also been extensively employed, not only for capturing time-averaged statistics, but also to allow capture of ensamble-averaged full-field data using trigger signals from simultaneous fluctuating surface pressure measurements. (Results from these latter measurements will be discussed in future publications.) Both a Dantec system and a TSI system were used; each employed the same New Wave Gemini $120 \mathrm{~mJ}$ Nd:Yag dual-fire laser to illuminate the field, usually in vertical planes aligned at various angles to the axial direction. The cameras and algorithms allowed final interrogation areas between $0.6 \mathrm{~mm}^{2}$ and $1.2 \mathrm{~mm}^{2}$, 
with analysis done using recursive algorithms designed to allow shifting of successive interrogation areas by an amount depending on the local velocity (Hart, 1999), to minimise errors. Validation of the resulting vectors led to rejection of at worst about $5 \%$ of the vectors - but usually far fewer. Typically, 1000 image pairs were obtained in every case; this was a compromise between minimising the statistical errors arising from a finite sample size whilst maintaining a reasonable total sampling time. With sampling rates of around $2 \mathrm{~Hz}$, the latter was typically around $500 \mathrm{~s}$. Seeding for both LDA and PIV measurements was provided either by a hydrosonic seeder or a standard smoke machine. Both yielded particle images of appropriate size (2-4 pixels in the PIV case). A particular problem arose in obtaining data very close to the cube surfaces, where reflective flare from the laser light sheet impinging on the surface led to additional noise in the data. There is some literature on this reflective flare problem (e.g. Wernet, 2005 and Shavit et al. 2007 and many references therein). In our experiments we reduced the resultant noise to acceptable levels by applying a non-reflective paint on the top surface and using an appropriate optical filter on the CCD camera. This made it possible to collect data at distances as close as about $2 \mathrm{~mm}$ from the surface.

\subsection{Numerical method}

The present numerical simulation has been carried out alongside the detailed experimental study, which was specifically designed to elucidate Reynolds number effects (providing a factor of 20 in Re at model scales with field data giving another order of magnitude increase in Re). That aspect of the work has been fully described by Lim et al. (2007). Additional velocity and pressure measurements and, in particular, PIV sections and integral measures of the flow structure have been obtained. This more extensive dataset allows us to assess various aspects of the LES computations - like the effect of adjusting the sub-grid model (SGM) or the rough wall treatment. Details of the basic computational approach are given below.

\subsubsection{Governing equations}

The governing equations used for large-eddy simulation are well known, but they are reiterated here, for completeness. We consider an incompressible fluid of kinematic viscosity $\nu$ in motion with kinematic pressure $p$ and velocity $u_{i}=(u, v, w), i \in 1,2,3$ along Cartesian axes $x_{i}=(x, y, z) \in \Omega$ within some domain $\Omega \in R^{3}$ aligned along the streamwise, spanwise, and vertical directions, respectively. In order to resolve the filtered velocity field $\hat{u}_{i}$ on a relatively coarse grid, we require scale-limited solutions of the Navier-Stokes equations on limited grid spacing, which is proportional to the specified filter width, say the grid size $\triangle$. The spatial filtering operation is indicated by a hat ( $)$, so that if $f(x)$ denotes some variable then the filtered variable is given by

$$
\widehat{f\left(x_{i}\right)}=\int G\left(\left|x_{i}-x_{i}{ }^{\prime}\right|\right) f\left(x_{i}{ }^{\prime}\right) d \Omega, \quad x_{i}, x_{i}{ }^{\prime} \in \Omega
$$

where $G \geq 0$ represents a filter kernel of width $\sim 2 \triangle$. The subgrid-scale (SGS) motions are eliminated by filtering the Navier-Stokes equations, which (with additional SGS stress modeling) yields the system of equations 


$$
\begin{aligned}
& \frac{\partial \widehat{u_{i}}}{\partial t}=P_{, i}+\left(-\widehat{u_{i}} \widehat{u_{i}}+\tau_{i j}-\frac{1}{3} \tau_{k k} \delta_{i j}+2 \nu S_{i j}\right)_{, j}+b_{i}, \\
& u_{k, k}=0, \\
& 2 S_{i, j}=\left(\widehat{u_{i, j}}+\widehat{u_{j, i}}\right), \\
& \tau_{i, j} \equiv-\left(\widehat{u_{i} u_{j}}-\widehat{u_{i}} \widehat{u_{j}}\right), \\
& E_{S G} \equiv-\tau_{k k} \geq 0, \\
& \tau_{i, j}-\frac{1}{3} \tau_{k k} \delta_{i j}=2 \nu_{s g} S_{i j}, \\
& \nu_{s g}=\max \left\{\left(c_{0} \triangle\right)^{2} D(\zeta)^{2} S-\nu, 0\right\},
\end{aligned}
$$

where $P=\widehat{p}-1 / 3 \tau_{k k}$ denotes the modified pressure, $b_{i}$ the kinematic body force per unit volume, $S_{i j}$ the symmetric part of the deformation rate tensor, $t$ the time, $\tau_{i j}$ the SGS stress tensor, and $E_{S G}$ the SGS kinetic energy; the negative trace of $\tau_{k k}$ follows from the positive filter kernel (see, Ghosal et al. , 1995). In the following we drop use of the hat for convenience unless explicitly required. We use the Smagorinsky (1963) (slightly modified) sub-grid model to compute the sub-grid eddy viscosity $\nu_{s g}$ using a Smagorinsky constant $c_{0}=0.1$; here $S=\left(2 S_{i j} S_{i j}\right)^{2}$, and $D(\zeta)$ is a wall damping function (Mason, 1994, $n=2$ ) defined in terms of $\zeta=l_{w} / \triangle$, where $l_{w}$ the nearest wall distance. We follow the usual LES practice and take $\triangle=\left(\triangle_{x} \triangle_{y} \triangle_{z}\right)^{1 / 3}$, where $\left(\triangle_{x}, \triangle_{y}, \triangle_{z}\right)$ is the finite difference grid interval. The rough wall boundary treatment is discussed in Section 2.2.3.

\subsubsection{Computational technique}

A multi-block computational domain was used, with the horizontal plane covering $-4<x / h<6$ axially and $-2.5<y / h<2.5$ laterally and subdivided into 50 unit-sized (side $h$ ) blocks, with this pattern repeated vertically up to $z / h=5$; the origin of the Cartesian coordinate system is at the bottom, spanwise centreline of the front face of the cube. The time-dependent Navier-Stokes equations, (4) above, were discretised using second-order finite difference methods in space and a second-order Adams-Bashforth method in time. As described in Section 2.2.1, the computational grid had a limited resolution $2 \triangle$ and the large eddy simulation technique employed (see for example the reviews by Mason, 1994, Lesieur and Metais, 1996 and more recently in a wind engineering context Murakami, 1997) retains explicitly only those scales resolvable on the grid while using the Smagorinsky (1963) subgrid model to account for the motions at scales smaller than $2 \triangle$. The grid was arranged so that the grid lines coincided with the boundaries of the cube and was therefore aligned normal to the mean flow direction. It had $320 \times 160 \times 160$ cells in the streamwise, spanwise and vertical directions respectively, giving about $\sim 10^{7}$ degrees of freedom and a (uniform) grid size of $\triangle=h / 32$. Further details of the numerical method used in the present work and the domain decomposition approach can be found in Thomas \& Williams (1997). The equations were solved using the complex geometry multigrid LES code CgLES using $\mathrm{C} / \mathrm{C}^{++}$and MPI message passing libraries, and ran on a 32 dual node Intel cluster. 


\begin{tabular}{|c|c|c|c|c|c|c|}
\hline Case & Domain size & Domain grid & Wall model typ & $u_{*} / U_{h}$ & $\beta$ & $\mathrm{n}$ \\
\hline 01 & & & Schumann & 0.059 & 1 & - \\
\hline 02 & $10 \mathrm{~h} \times 5 \mathrm{~h} \times 5 \mathrm{~h}$ & $320 \times 160 \times 160$ & corrected & 0.057 & 0.3 & - \\
\hline 03 & & & higher order & 0.058 & 0.25 & 2 \\
\hline Exp. & $9 \mathrm{~m} \times 0.6 \mathrm{~m} \times 4.5 \mathrm{~m}$ & - & mesh grid & 0.059 & - & - \\
\hline
\end{tabular}

Table 1

Case details for the channel inflow simulation.

\subsubsection{Surface roughness boundary conditions}

Surface roughness is usually treated with a synthetic wall model in which numerical solution of the very small scales of the flow around individual roughness elements is replaced by assumptions about the local mean and fluctuating velocity distributions based on universal profiles for rough surfaces. In the present LES simulation three variations of the Schumann wall treatment were used; these are now discussed briefly.

Firstly, we applied a Schumann-type wall model on the wall boundaries, following the original development by Schumann (1975). It is a wall model in the sense that the wall stresses are determined directly from an algebraic model, by assuming that they are in phase with the velocity at the first off-wall grid point and that the deviation from their mean is proportional to the deviation of the velocity from its long time mean value. Many improvements to this basic model have been proposed and tested. One of these (Thomas $\&$ Williams, 1999) is a correction of the fluctuating part to reduce the anomalously large wall dissipation when the scheme is applied to a (rough) atmospheric sub-layer (ASL) inflow. This correction involves splitting the mean, $U$, and fluctuating components, $u-U$, of the velocity so that

$$
\frac{\left(\tau_{x}, \tau_{y}\right)}{u_{*}^{2}}=\frac{1}{u_{a}}(U, V)+\frac{\beta}{u_{a}}(u-U, v-V)
$$

where the new parameter $\beta \leq 1$ is a damping factor applied to the fluctuating part and $u_{a} / u_{*}=\gamma^{-1}=\log _{e}\left(z / z_{0}\right) / \kappa$ introduces a Jensen number dependence; if $\beta=1$ the scheme reduces to the original Schumann form. In essence, the steady flow sees a rough wall whereas the unsteady flow sees a less rough wall. The energy dissipation per unit area due to the boundary conditions is $\beta \gamma\left(\sigma_{x}^{2}+\sigma_{z}^{2}\right) u_{*}$. With $\beta=1$, this is acceptable for smooth walls but becomes unacceptably large for the very rough surface required here; the computed turbulence levels near the wall were far too low as was the overall turbulence intensity. One solution considered was to re-inject the turbulent kinetic energy using the stochastic backscatter subgrid model of Mason and Thomson (1992). However, the magnitude of the random forcing required by the model would lead to an unsteady velocity field with a significant stochastic component; it was clear that while the statistics of this flow would be acceptable the unsteady dynamics would probably be too random and therefore not properly representative of the coherent structures expected to be important in the dynamic loading on the body. In the present work we set $\beta=0.3$ as a second wall condition to match the experimentally observed turbulence intensity levels, so that the unsteady flow sees a rather smoother wall. This value was tested by Thomas \& Williams (1999) and they found that $\beta$ will control the magnitude of the flow fluctuation (i.e. $\sigma_{x}, \sigma_{z}$ etc.) near the surface and could be fine-tuned to match particular experimental data. Another wall model used in this study was one suggested by Xie et 
al. (2004), which considers a non-linear effect in the amplitude of flow fluctuation and chooses a higher-order damping factor in the wall model. They also pointed out that a second order-damping factor with the $\beta=0.25$ appeared to be optimal. The second order wall model can be described as follows:

$$
\begin{aligned}
\frac{\left(\tau_{x}, \tau_{z}\right)}{u_{*}^{2}} & =\frac{1}{u_{a}}(U, V) \\
& +\frac{\beta}{u_{a}} \frac{\left((u-U)^{2},(v-V)^{2}\right)^{n / 2}}{u_{*}^{2}}(u-U, v-V), n \geq 0 .
\end{aligned}
$$

Table 1 summarises the three boundary condition cases. It turned out that, in fact, all approaches yielded very similar results, as will become clear in due course.

It is worth mentioning that in none of these approaches is a classical log-law behaviour employed for conditions at the first grid point away from the wall. This is often done, and practically always in RANS methods of course, but it would be particularly inappropriate in regions where one might not expect log-law behaviour in the near-surface region. Such regions certainly include the separated regions just upstream and just downstream of the cube, although it can be argued that there even the near-surface flow is dominated by larger-scale motions arising from shedding of vorticity from the cube and the precise form of the boundary condition is thus not likely to be important. The same could be argued for the surfaces of the cube itself, particularly the top surface above which there is an unsteady separated region. There is no reason to suppose that the above approaches would cease to be physically reasonable in these 'more difficult' regions of the flow.

\subsubsection{Inflow boundary layer generation}

In LES computations of bluff body flows it is necessary to generate inflow turbulence (i.e. the inflow boundary conditions) which properly represents the turbulent flow in which the body is immersed, analogous to the wind environment of a building. The turbulence must reproduce, at least, the appropriate degree of wind shear, the level of turbulent intensity and the integral scale of the turbulence. Turbulent intensity, for example, is important because the way in which the flow responds to the presence of the obstacle depends, amongst other things, on how high external turbulence levels are compared with the turbulence generated directly by the obstacle. The ratio of the integral length scale of the upstream flow to the obstacle size is perhaps less significant; Lim et al. (2007) followed Melbourne (1980) in arguing that matching the small-scale turbulence levels is much more important in determining (for example) peak pressures on the body. There are essentially two methods of generating the necessary inflow: one is statistical, whereby a sequence of random numbers is created and then filtered to yield the appropriate statistical properties and spatial correlations, and the other is to perform a separate 'precursor' simulation of a wind environment and sample the inflow data directly from this. The second method has the desirable property that the generated inflow should naturally contain physically realistic coherent structures, without these having to be produced artificially; this is the method adopted in the present work. A similar method was used by Nozawa \& Tamura (2002) in their computations of flow over a half-cube. However, they used sets of individual blocks in their precursor simulation to generate suitable rough-wall boundary layers but then, as noted earlier, used smooth walls in the 
half-cube computational domain; the influence of this effective sudden change in surface roughness at the start of the model domain is not clear. Recently a new, very efficient version of the alternative 'digital filter' approach has been demonstrated to be effective for rough-wall, urban-types of flows (Xie \& Castro, 2008), but this was not available at the time the present computations were done.

The turbulent inflow for the bluff body simulation was generated using a precursor LES of a wind environment, in which the fluctuating velocity field $(u, v, w)$ at the location of its outflow boundary was sampled at each time step and saved in a wind database. The full details of the precursor simulation methodology are given by Thomas \& Williams (1999). In this simulation periodic boundary conditions ensure that the turbulence is developed by recycling. The computation was run using a domain height identical to that used for the subsequent computation of the cube flow, until a fully developed boundary layer was established, after which the outlet field was used as the inlet field for the bluff body computation. For the latter, convective outflow boundary conditions based on an upwind treatment of the convective terms were applied on the outflow faces of the computational domain. The convective velocity was taken as the local velocity at the upstream face of the boundary cell, and the velocity gradient was evaluated using a first-order upwind difference applied to the boundary cell. This approach had been used previously by one of the writers for computation of laminar vortex shedding from a 2D square cylinder and was found to perform satisfactorily. For both the precursor and the bluff body computations a free-slip upper boundary condition was used and the surface roughness conditions were identical and modelled as described in $§ 2.2 .3$.

\section{Results and discussion}

\subsection{The approaching turbulent boundary layer}

Results from the separate inflow (precursor) simulation were sampled after conditioning for approximately $100 h / u_{*}$ - significantly longer than that in Lim \& Thomas (2005) thus allowing for better discrimination between the different wall treatments. The samples were averaged spatially over the periodic domain and over the period $50 h / u_{*}$. Velocity and turbulence profiles normalized using $u_{*}$ are shown in Figures 2 and 3. Note that the mean velocity profile in Figure 2(a) obtained from the wind tunnel was actually for a Reynolds number $\left(R e_{h}=U_{h} h / \nu\right)$ range $1.86 \times 10^{4} \leq R e_{h} \leq 7.31 \times 10^{4}$, in comparison with the current computed profile at $R e_{h}=2.0 \times 10^{4}$. However, the experiments showed no profile variation with $R e$. The mean velocity fitted a standard log-profile up to at least $z=3 h$, so that the cube was fully submerged within the log-law region of the turbulent boundary layer. Figure 2(b) shows the corresponding Reynolds shear stress, $-\overline{u w}$, profile. Here, and henceforth, we employ the common notation of lower case symbols denoting fluctuating quantities, with dashed symbols denoting rms values (so $u^{\prime} \equiv \sigma_{u} \equiv \sqrt{\overline{u^{2}}}$ ). The profiles indicate that the simulations are sufficiently converged and that the results for all wall models appear similar - indeed, virtually indistinguishable - and in reasonable agreement with the wind tunnel measurements. The constant pressure gradient forcing used in the computations implies a nearly linear variation of shear stress with height, whereas in the wind tunnel it reduces more rapidly above $z=1.5 \mathrm{~h}$. 


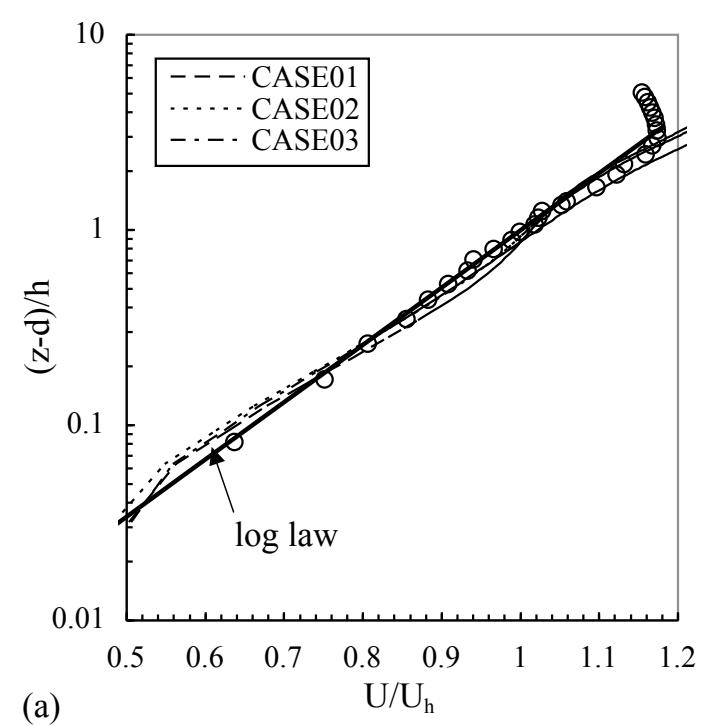

(a)

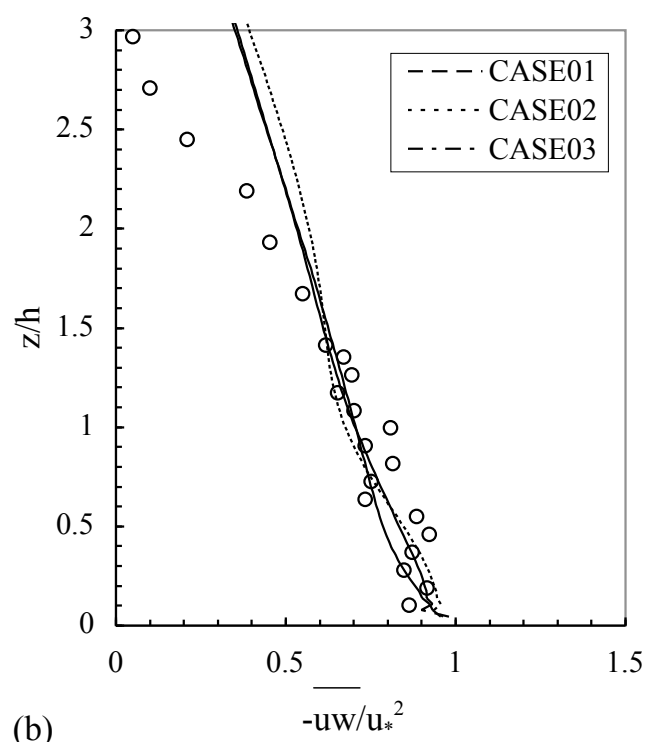

(b)

Fig. 2. Mean velocity (a) and shear stress (b) profiles. Symbols (circles) are the experimental data. The straight, bold solid line in (a) is the log-law with $z_{0}=0.09 \mathrm{~mm}, u_{*} / U_{h}=0.0592$, and the fainter lines are the computations using the three wall approaches - virtually indistinguishable.

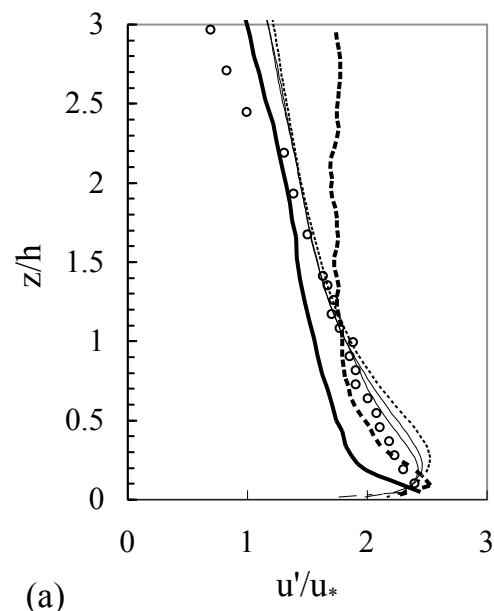

(a)

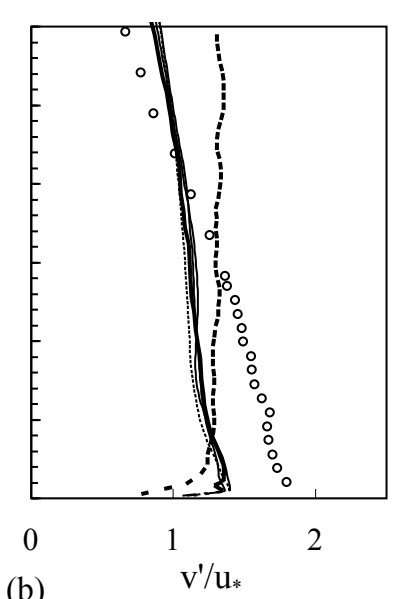

(b)

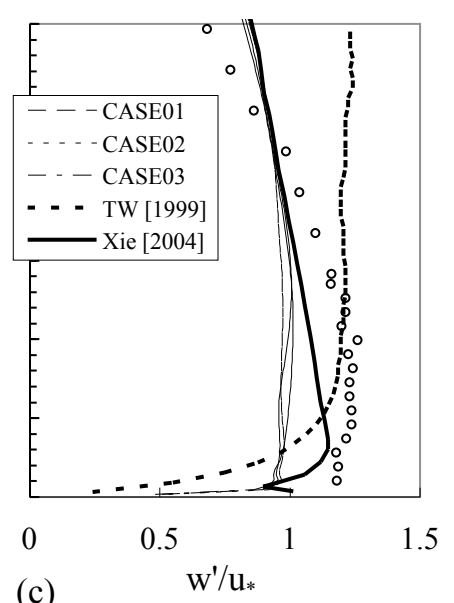

(c)

Fig. 3. Axial (a), spanwise (b) and vertical (c) rms turbulence non-dimensionalised by $u_{*}$. Symbols (circles) are the experimental data. Note that the lighter lines refer to the three present wall function approaches and are largely indistinguishable.

Figure 3 shows the axial rms (i.e. $u^{\prime}, v^{\prime} \& w^{\prime}$ ) profiles normalised by $u_{*}$ and also the comparison between our calculated data and previous numerical studies - Xie et al. (2004) and Thomas \& Williams (1999). The computed streamwise turbulence $\left(u^{\prime} / u_{*}\right)$ broadly matches that found in the experiments. Below $z / h \leq 1$ CASE01 data are a little closer 


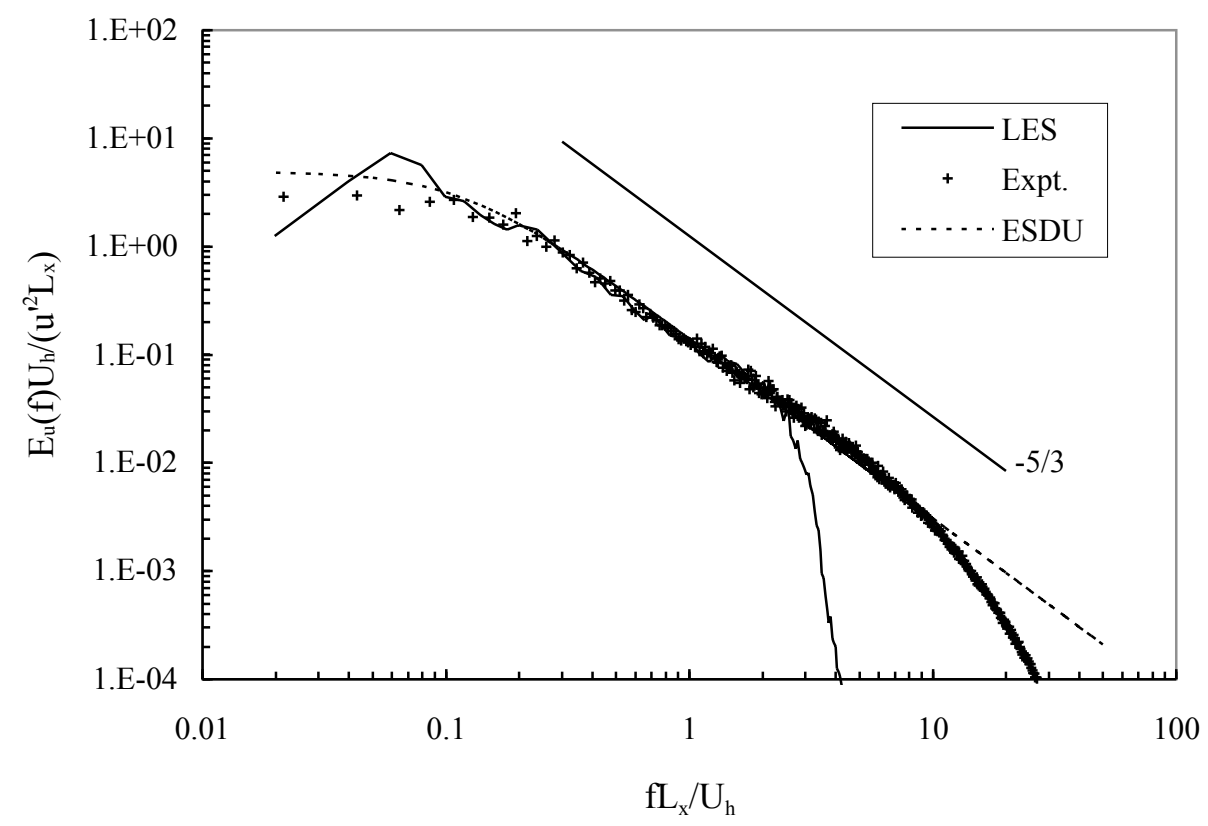

Fig. 4. Spectra of the axial turbulence component at $\mathrm{z}=\mathrm{h}$.

to the measured data. However, note that a 20-30\% underprediction on the vertical and spanwise turbulence levels is observed and the mean velocity is also slightly overpredicted. We believe that this may be due to imperfect wall functions and is not related to the periodic boundary condition used in the inflow generation process. It seems not to depend significantly on which wall approach is used. This defect has been reported in previous studies and is a common feature of simulations using finite-difference methods on relatively coarse meshes (see Rai \& Moin, 1993). For the present purposes, the differences were considered acceptably small and it was thus concluded that the upstream turbulence is a satisfactory representation of the wind tunnel boundary layer, virtually independent of which of the three surface conditions are used. This is further confirmed by spectral and autocorrelation data which are presented next. For all the remaining LES data presented here the CASE01 (Table 1) was used.

Figure 4 shows typical longitudinal-velocity spectra, $E_{u}(f)$, obtained at $z=h$ and normalised using $U_{h}$ and $L_{x}$ (which should give collapse in the inertial region). At least one decade of inertial sub-range with slope $-5 / 3$ is seen in the spectrum obtained from both the calculation and the wind tunnel measurements. There is good agreement in the spectra plotted in these terms, although note that the combination of a finite domain length in the computations $(10 h)$ and turbulent forcing from the upstream grid arrangements in the wind tunnel lead to an integral length scale in the experiment which is somewhat larger than in the computations, consistent with the elevated levels of vertical intensity. The wind tunnel spectrum was also fitted to the ESDU spectrum (ESDU, 1985). Although the LES data in the inertial subrange also appear to collapse quite well with the ESDU spectrum, there is a rapid drop beyond about $f L_{x} / U_{h}=2.5$, which is 


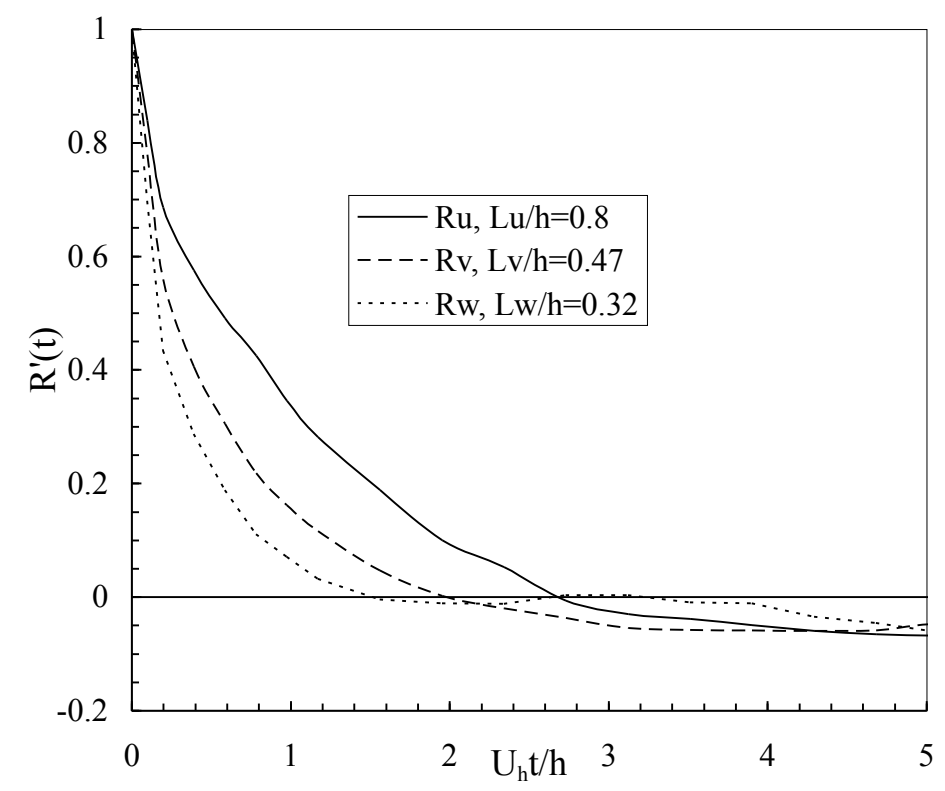

Fig. 5. Normalised autocorrelation functions at $z=h$. Ru (in legend) refers to $R_{u u}$, etc.

Table 2

\begin{tabular}{cccccccc}
\hline Case $U / u_{*}$ & $u_{r m s} / u_{*}$ & $v_{r m s} / u_{*}$ & $w_{r m s} / u_{*}$ & $-\overline{u w} / u_{*}^{2}$ & $h / z_{0}$ & $L_{x} / h$ \\
\hline 01 & 16.8 & 1.82 & 1.21 & 1.04 & 0.71 & & \\
02 & 17.4 & 1.86 & 1.16 & 0.99 & 0.68 & 600 & 0.8 \\
03 & 17.1 & 1.82 & 1.24 & 1.03 & 0.70 & & \\
\hline Exp. 16.94 & 1.88 & 1.49 & 1.26 & 0.81 & 890 & 1.63
\end{tabular}

Salient parameter values at $z=h$.

due to the limited grid resolution.

The autocorrelation functions of the three velocity components at $z=h$ are shown in Figure 5 . They are defined in the usual way $-R_{u u}(t)=\overline{u\left(t_{0}\right) u\left(t_{0}+t\right)}$, for example, with the $R^{\prime}(t)$ label on the abscissa denoting normalisation by the appropriate mean-square fluctuating velocity. All the correlations are small for a non-dimensional time, $U_{h} t / h$, greater than about unity, thus confirming that the computational time (about $32 h / U_{h}$ ) is sufficiently long. Nonetheless, extending the time axis leads to a noticeable periodicity in each $R^{\prime}(t)$, corresponding to the finite domain length of 10h. The area under the $R_{u u}$ curve to the point of zero $R_{u u}, T_{x}$ say, yields an (axial) integral length scale of about $L_{u}^{x}=0.8 h$ (using Taylor's hypothesis so that $L_{u}^{x}=U_{h} T_{x}$ ), which is somewhat lower than that of the wind tunnel flow, as noted earlier. Table 2 summarises the salient parameter values for the upstream boundary layer. 


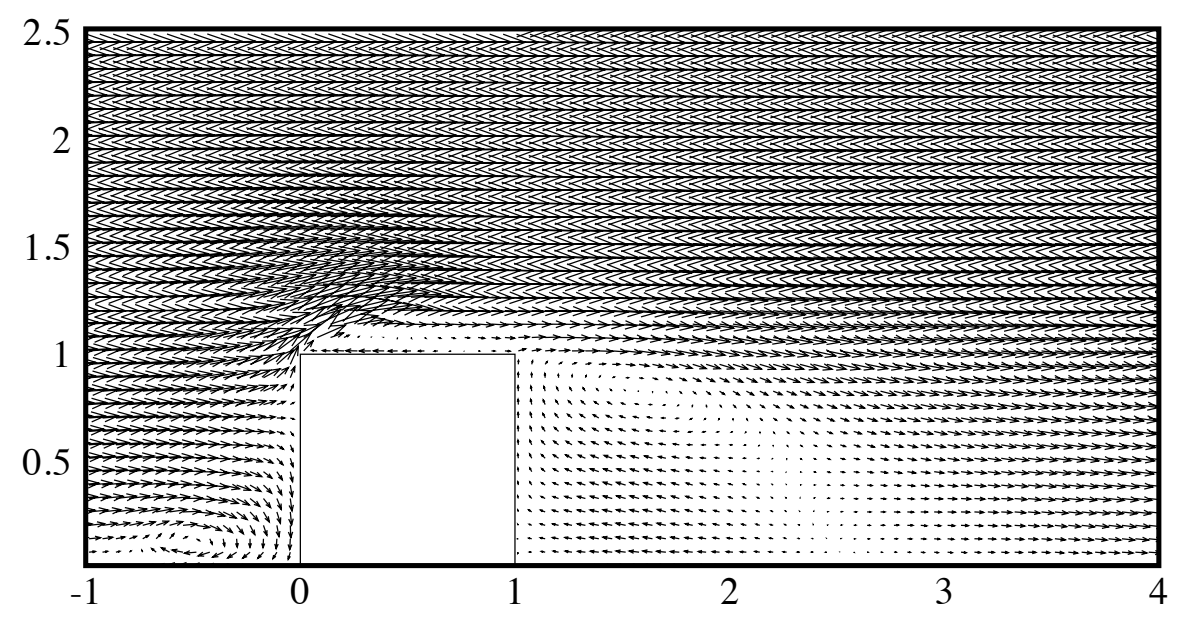

Fig. 6. Mean velocity vectors of $(u, w)$ in the $x-z$ plane through a cube. Axis numbers here and in figs. 7-9 are values normalised by $h$.

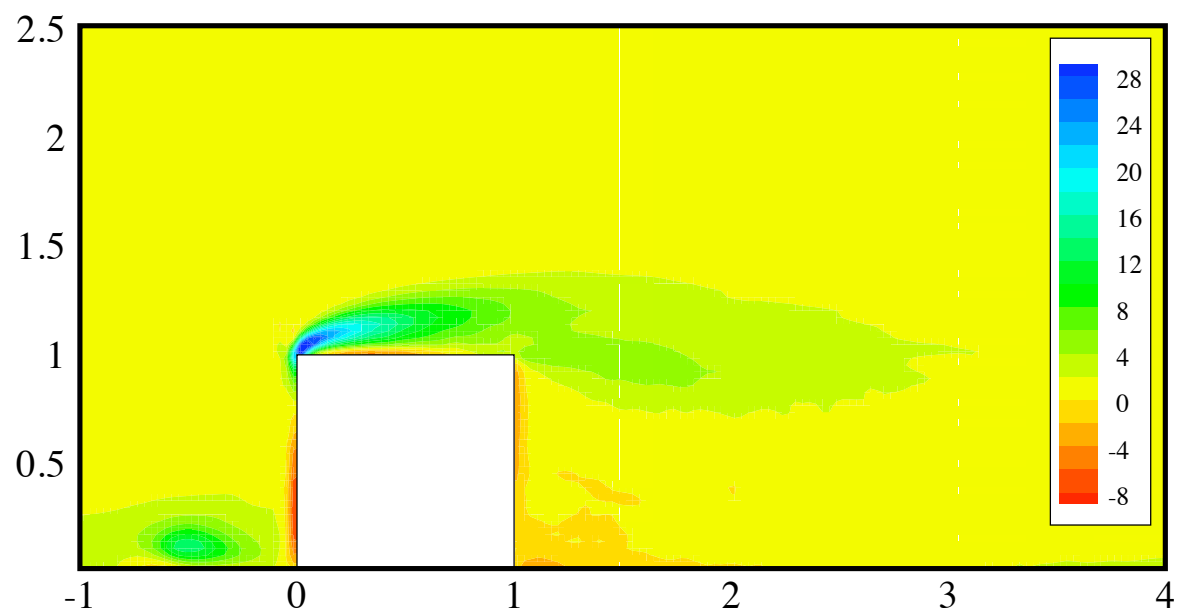

Fig. 7. Contours of mean vorticity $\omega_{y}$ in the $x-z$ plane through a cube.

\subsection{Overall flow around the cube}

We present first some general views obtained from the LES data, which characterise the overall structure of the flow around the cube. Figures 6 and 7 show, respectively, the timeaveraged vector $(u, w)$ and vorticity field $\left(\omega_{y}\right)$ around the cube along the central section $(x-z$ plane) from the LES calculation. The usual large recirculation region associated with the horseshoe (or 'necklace') vortex is evident on the surface just upstream of the cube and its center is located around $x / h \approx-0.5, z / h \approx 0.1$. Above this circulation region, a stagnation point on the front face of the cube is clearly observed at $z / h=0.73$. On the top surface, the separated region can be identified, with its attachment point around $x / h=0.75$. In addition, the downstream attachment in the near-wake occurs 


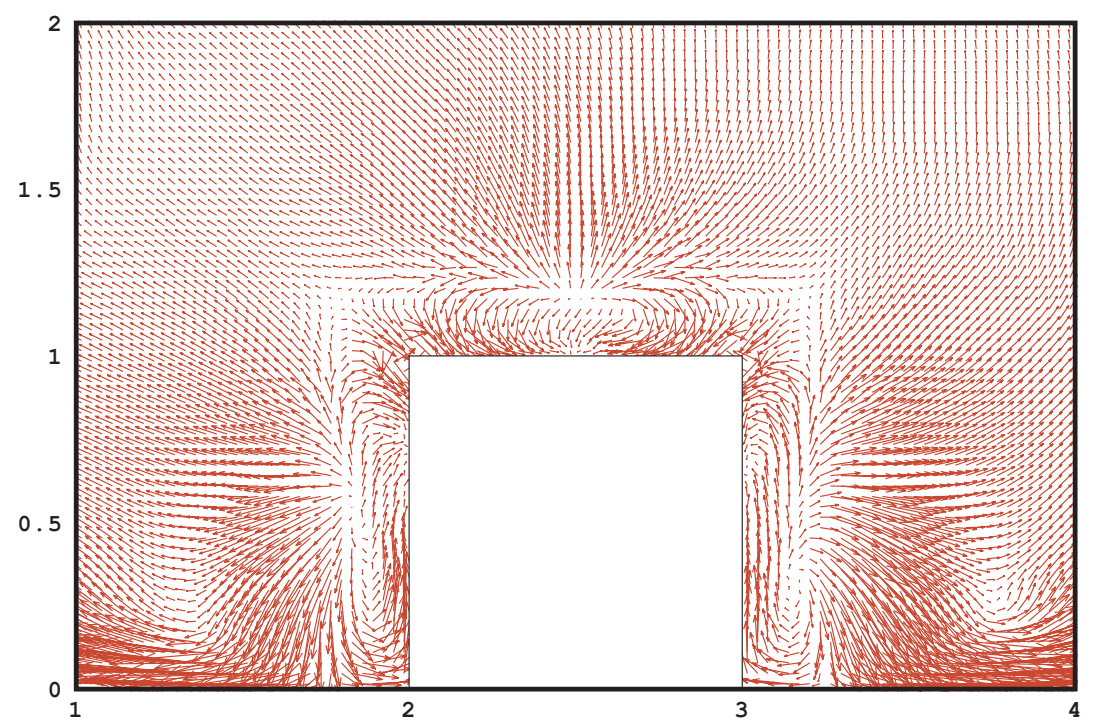

Fig. 8. Mean velocity vectors of $(v, w)$ in the $y-z$ plane through a cube.

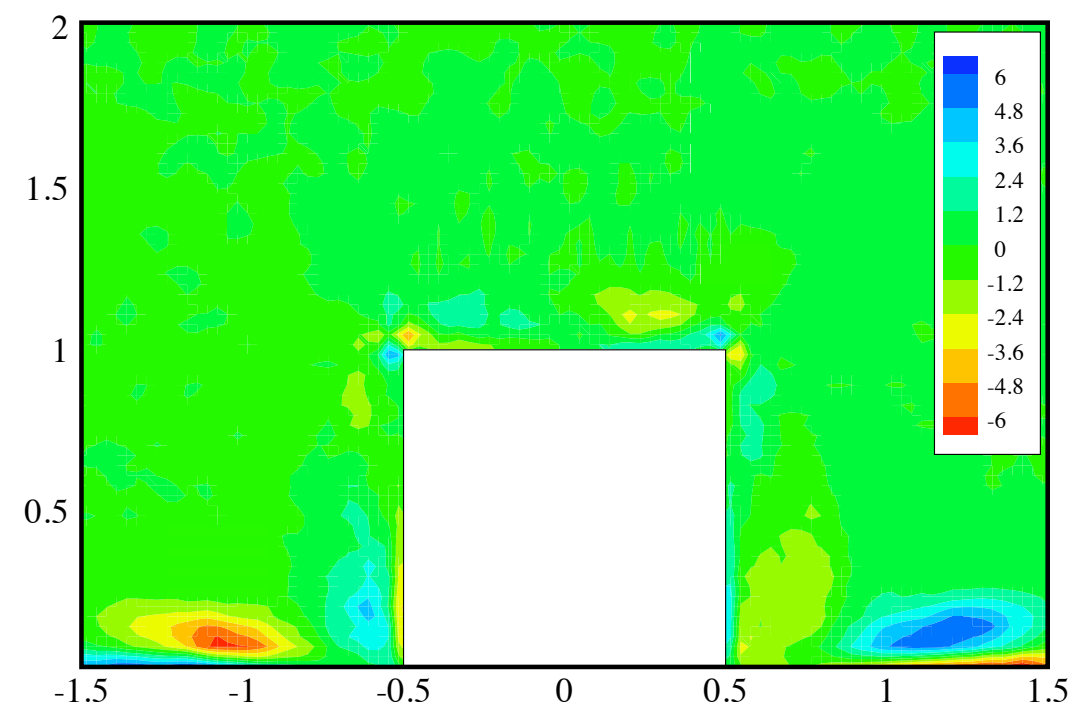

Fig. 9. Contours of mean vorticity $\omega_{x}$ in the $y-z$ plane through a cube.

around $1.56 h$ from the back of the cube. Plots of the cross-stream velocity and axial vorticity are shown in Figures 8 and 9. Note, in figure 8, the strong convergence of the cross-flow in the region around $0.5 h$ outside the side faces and $0.5 h$ above the surface; this is caused by the 'legs' of the horseshoe vortex, which are best identified by the high vorticity regions just above the surface, seen in figure 9.

Figure 10 shows 3 -dimensional iso-surface contours of pressure and vorticity. In the figure, the blue, the dark green and the green surfaces denote the positive and negative 


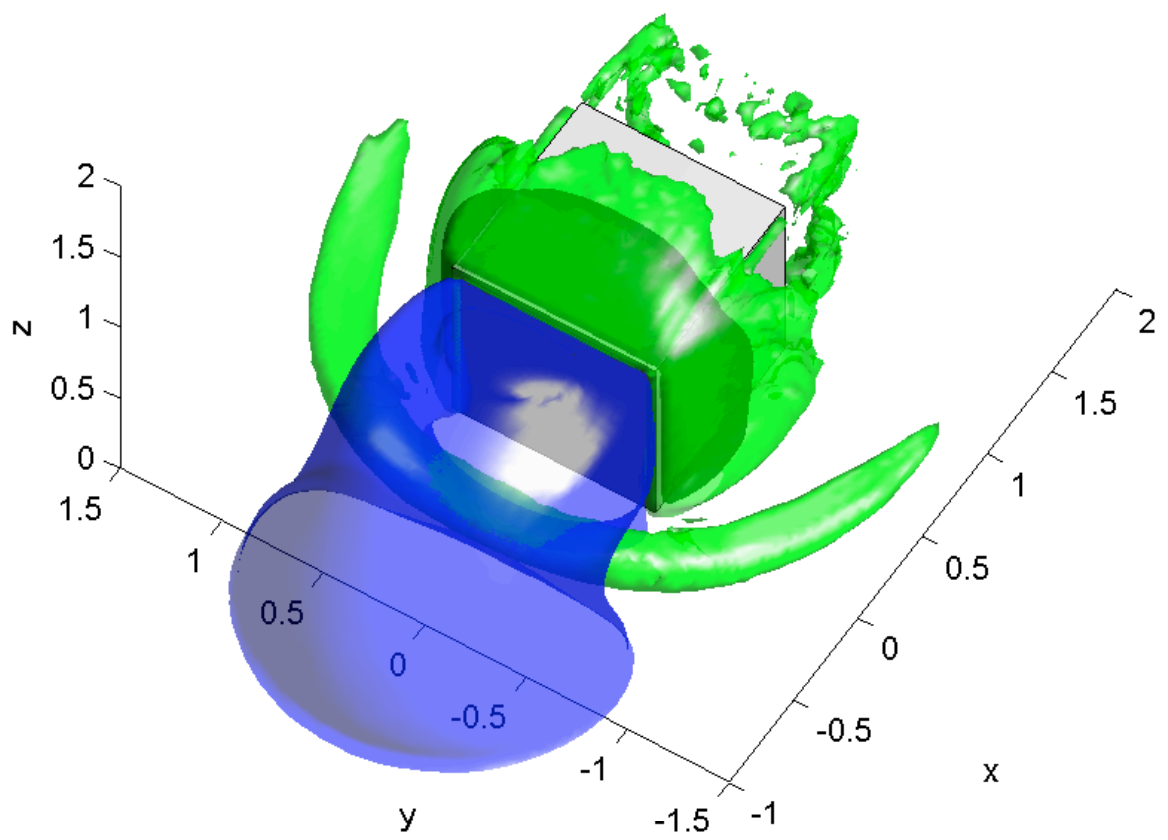

Fig. 10. Isosurface vortex structures and pressure distribution around the cube. The blue \& dark green colours indicate $3-\mathrm{D}$ contours of positive and negative pressures, with $C_{p}$ values of 0.06 and -0.9 , respectively. The light green contour denotes the topological vortex indicator $\Pi=-5$.

pressure, and the geometrical vortex indicator $(\Pi)$, respectively. The latter is defined as $\Pi=-L_{i j} L_{j i}$ where $L_{i j} \equiv \frac{\partial u_{i}}{\partial x_{j}}$ and is a measure of the regions of flow dominated by rotation rather than shear or stretching. In addition to the extent of the positive and negative pressure regions, the horseshoe vortex is very apparent. All these visualisations indicate a flow with the qualitative features expected on the basis of the existing literature. They are all seen, for example, in the cube-in-a-channel LES data of Shah \& Ferziger (1997) or, indeed, the more recent DNS data of Yakhot et al. (2006) for the same flow. Proper quantitative assessment of the adequacy of the computations, however, requires more detailed consideration of, for example, specific surface pressures and the near-cube velocity field and it is to these that we now turn.

\subsection{Details of cube flow}

\subsubsection{Mean and turbulence velocities}

We consider first the velocity field near the cube. Figures 11 to 15 present vertical profiles of the mean velocity, intensity and shear stress above the axial centreline of the cube, comparing the LES data with those measured using PIV and (in the leading edge region) LDA. The vertical gridlines along the $\mathrm{x}$-axis are equally spaced to delineate the spatial growth of the wind flow over the cube. In order to identify the clear flow variations around the separation region and compare the differences between the wind tunnel and LES data, the figures labelled (b) are magnified views of the leading-edge region. 

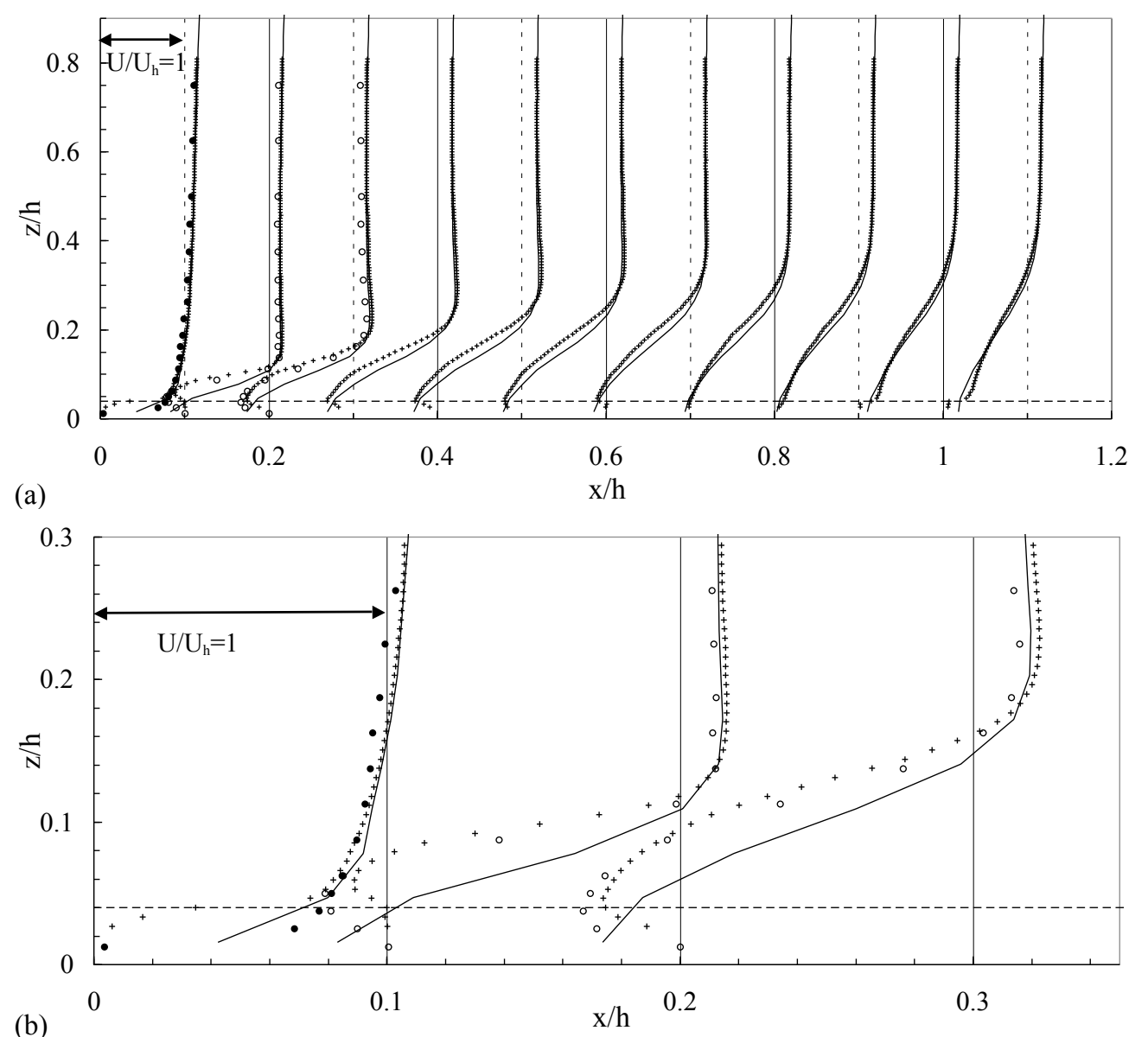

Fig. 11. Streamwise velocity profiles over the cube. Solid line is the present LES simulation and the symbols are the corresponding averaged values measured by PIV $(+)$ and LDA (o, open or solid). (b) is a magnified version of the first three positions shown in (a). The horizontal dashed lines are at $z=3$ $\mathrm{mm}$.

Figure 11 shows that the flow exhibits a typical separation at the leading edge $(x / h=$ $0.0)$ and an attachment region around three quarters of the distance along the top surface $(x / h=0.75)$. Note that there exists a discrepancy amongst the results within the circulation region behind the leading edge. In particular, the PIV and LDA results agree reasonably well at $x / h=0.2$ (see fig.11b), but the LES data are significantly higher close to the surface and this over-prediction is reflected in the data further downstream (fig.11a). This is almost certainly an LES spatial resolution issue (recall that the grid size was $h / 32$ ); finer grids would be required to resolve this region accurately. However, it is interesting that the 'cube in a channel' flow computed by Shah (1998) which, compared with the present case, was more finely resolved very near the surfaces but less resolved elsewhere, had significant discrepancies in the peak negative axial velocity in 


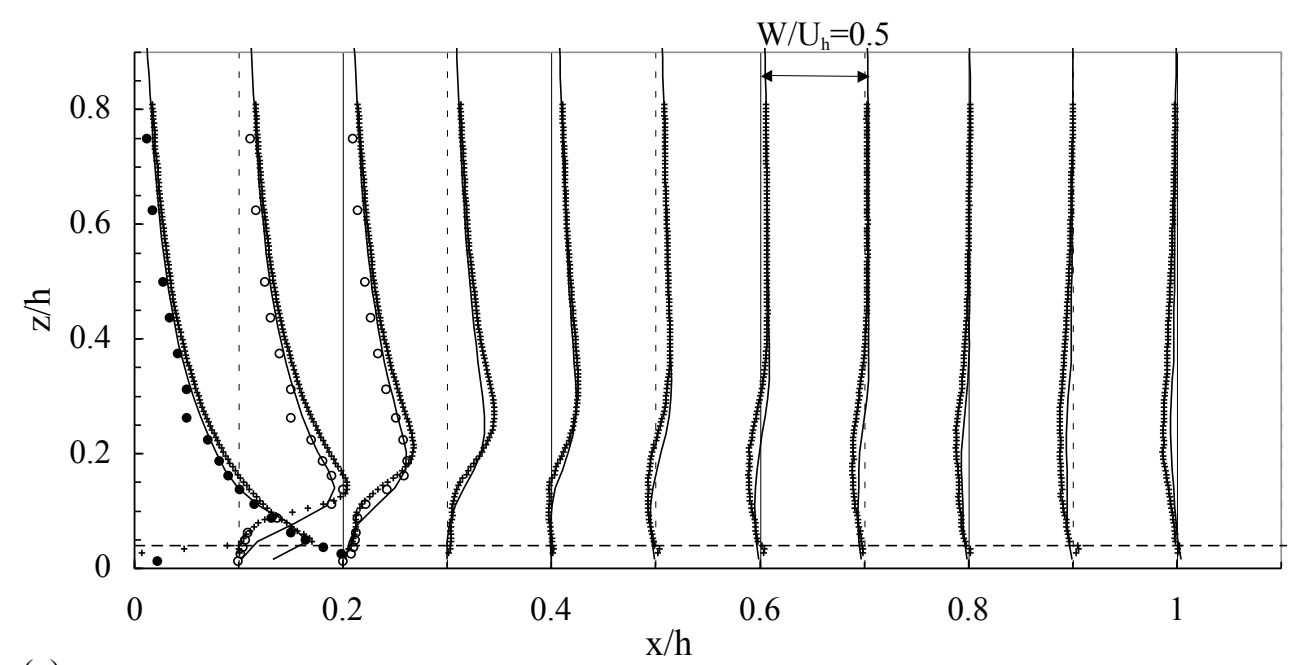

(a)

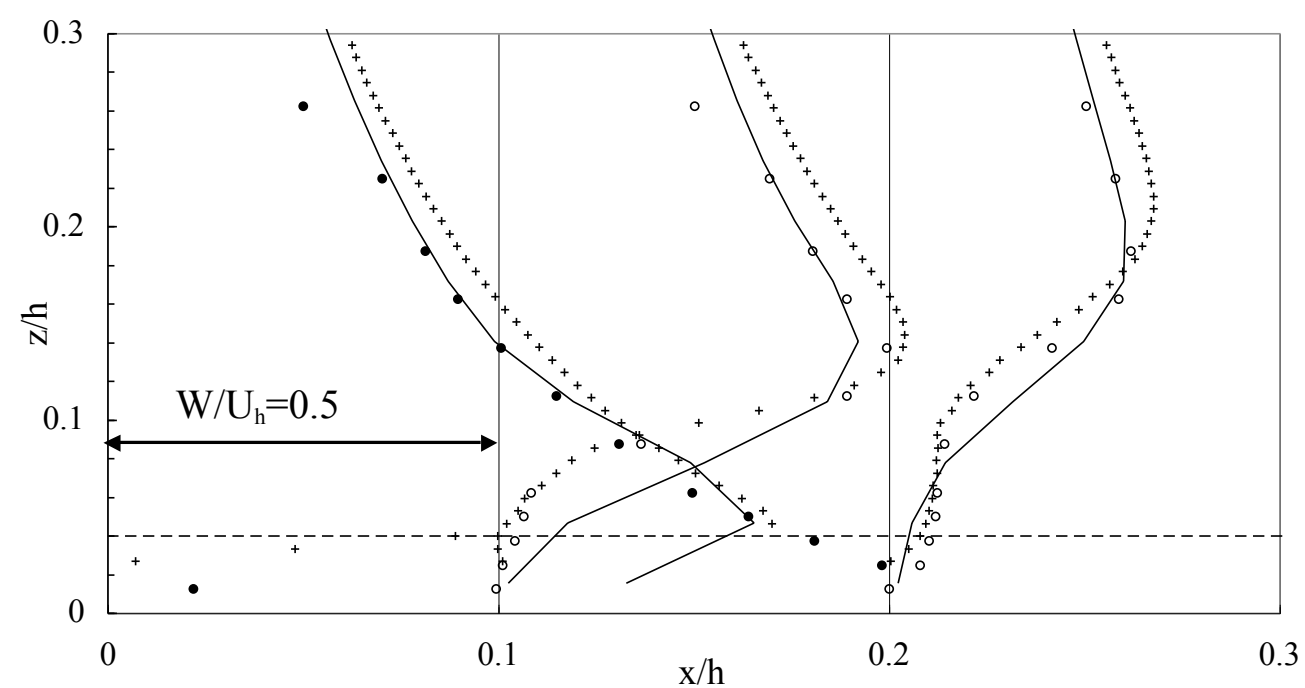

(b)

Fig. 12. Vertical velocity profiles over the cube. Symbols as in Figure 11.

the separated region on top of the cube.

Another interesting point arises at the leading edge $(x / h=0)$, where $U / U_{h}$ profiles from the LDA and the LES agree reasonably well, whereas the PIV results are significantly lower close to the top surface of the cube. This is particularly noticeable within about $3 \mathrm{~mm}$ (denoted by the dashed lines in figs.11-15), where PIV data are not always consistent with the LDA data. Since the PIV interrogation area size is, at its smallest, about $0.6 \mathrm{~mm} \times 0.6 \mathrm{~mm}$ (i.e. about $0.008 h$ square), the very high velocity shear near the surface, particularly near the leading edge, leads to a degree of inaccuracy in the 

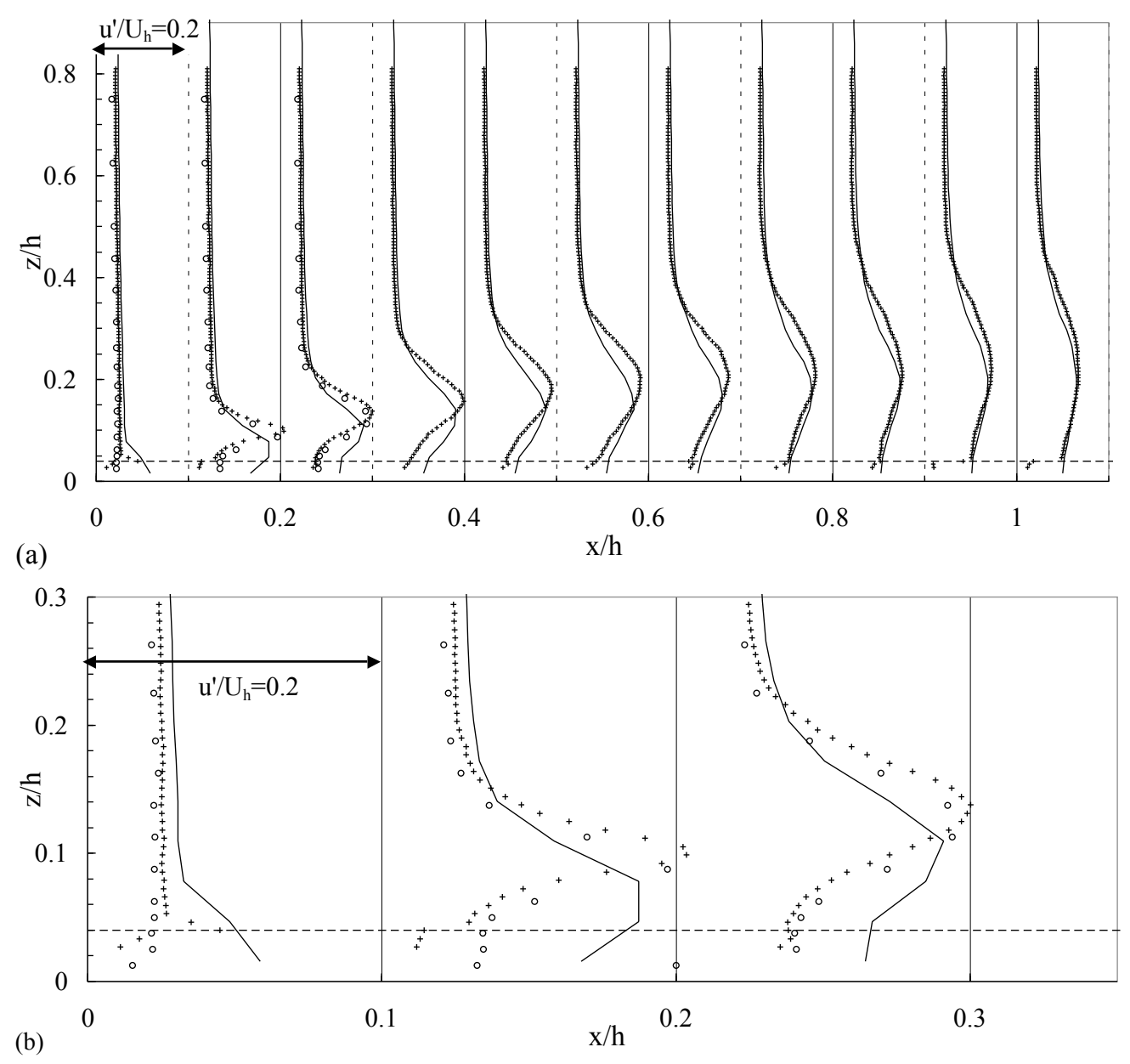

Fig. 13. Axial turbulence profiles $\left(u^{\prime} / U_{h}\right)$ over the cube. Symbols as in Figure 11 .

PIV data. This is very clear at $x / h=0.1$ (fig.11b), where the PIV data fail to capture either the strength or the thickness of the very thin reversed flow region there. Likewise, the LES data shows a much thinner separated region. The magnified profile of $W / U_{h}$ in fig. $12 \mathrm{~b}$ emphasises the high shears near the leading edge. Note, in particular, the very high vertical gradient of $W$ at $x / h=0$, indicated by the two LDA data points closest to the top surface, which is captured by neither the PIV nor the LES data. It is worth noting that Lim et al. (2007) argued that in these near-surface regions the typical spatial resolution of the PIV (normalised by $h$ ) was actually very similar to that of the sonic anemometers used in the related field experiments at Silsoe, so the latter showed similar differences from the LDA data, which are more highly resolved; this is clarified later.

Figures 13 to 15 show the rms turbulence and shear stress profiles $\left(u^{\prime} / U_{h}, w^{\prime} / U_{h}\right.$ and $-\overline{u w} / U_{h}^{2}$ ) over the cube. Overall, there is reasonable agreement between the experimental and computational data, but again there are significant differences near the leading edge. 


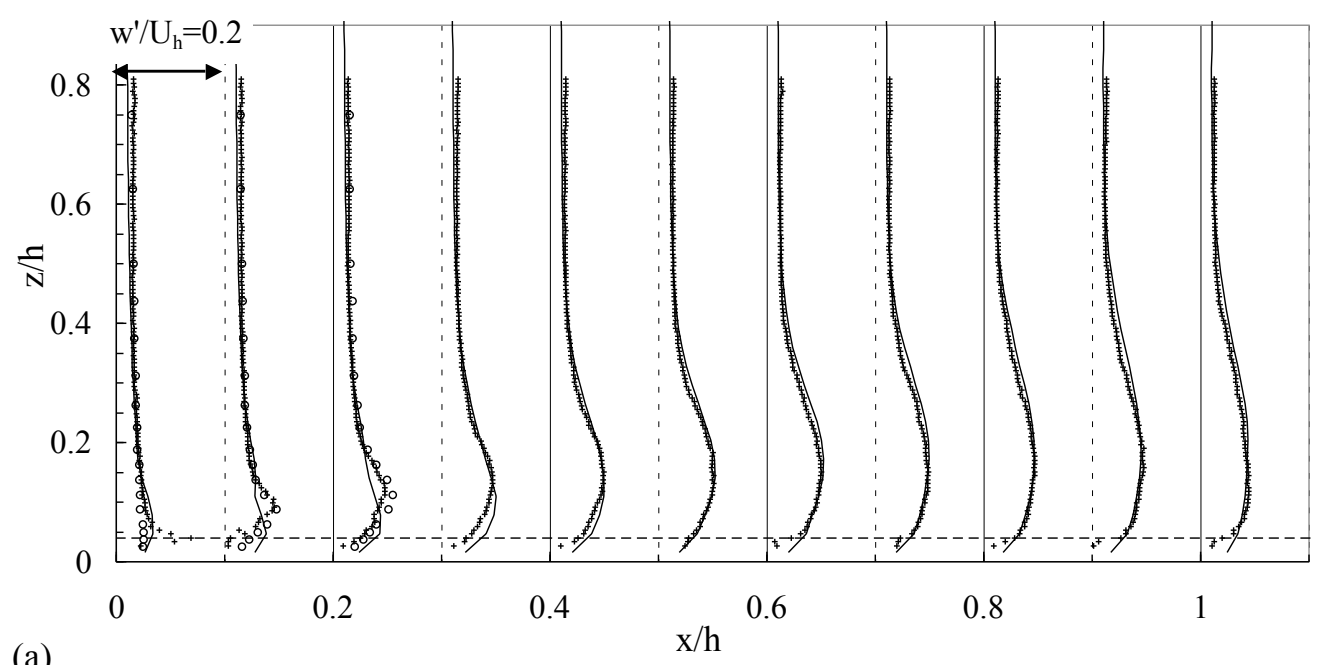

(a)

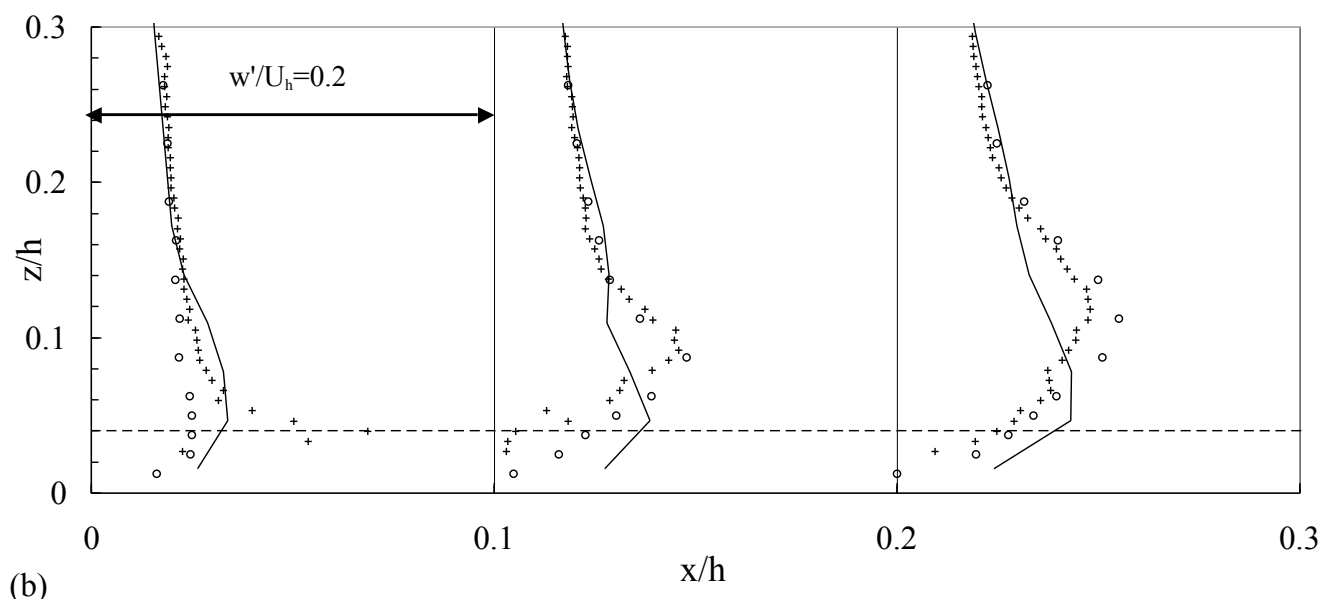

Fig. 14. Vertical turbulence profiles $\left(w^{\prime} / U_{h}\right)$ over the cube. Symbols as in Figure 11.

Note, for example, that the peak axial turbulence levels occurring near the edge of the body appear at different heights - at $x / h=0.1, z / h \approx 0.1$ (LDA, PIV) and 0.06 (LES), and at $x / h=0.2, z / h \approx 0.13$ (LDA, PIV) and 0.11 (LES). Again, the discrepancies immediately above the surface are most probably due to inadequate LES grid resolution for the high shear flow in this region. Nevertheless, the comparisons further downstream are quite good. The shear stress profiles, fig. 15, have peaks of different sign close to the top surface and further downstream, with a single negative peak for $x / h>0.2$, consistent with the sign of the mean velocity gradient there. Further upstream towards separation at $x / h=0$, the mean flow direction is not in the axial $(x-)$ direction (the boundary layer which separates at the leading edge is initially oriented vertically) so the individual stresses have less meaning in the basic $x-z$ coordinate frame. 


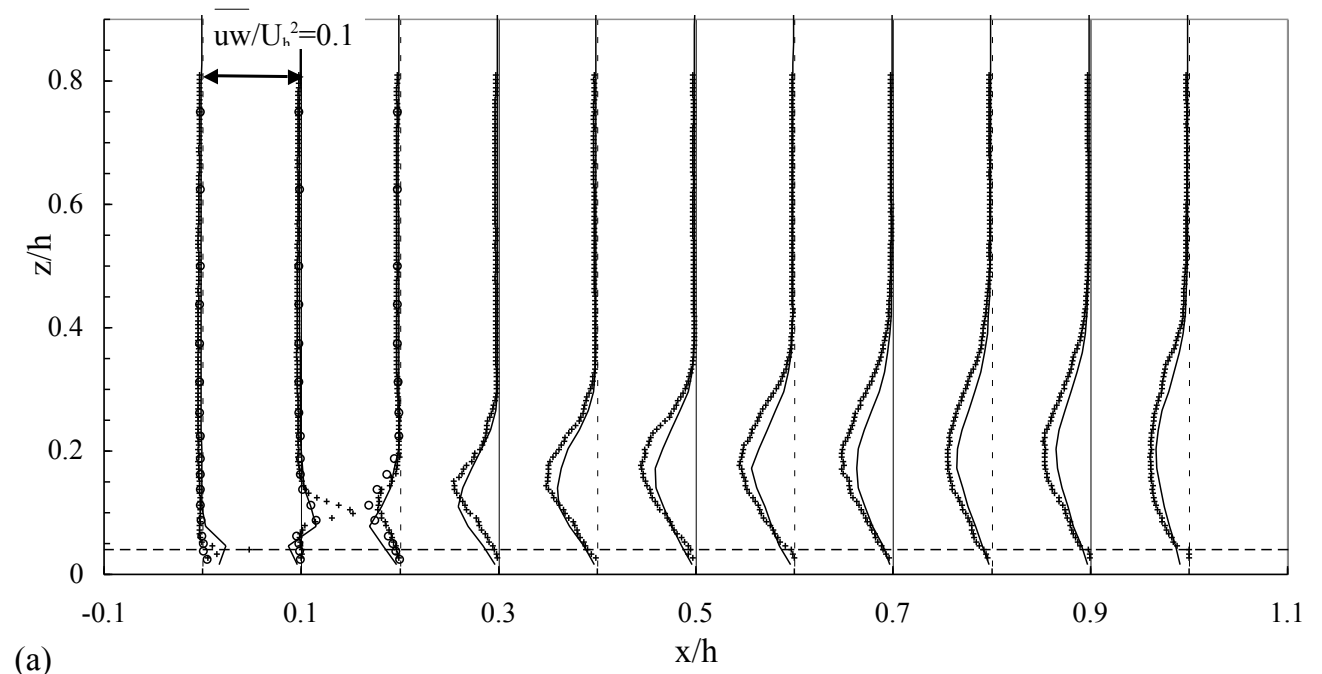

(a)

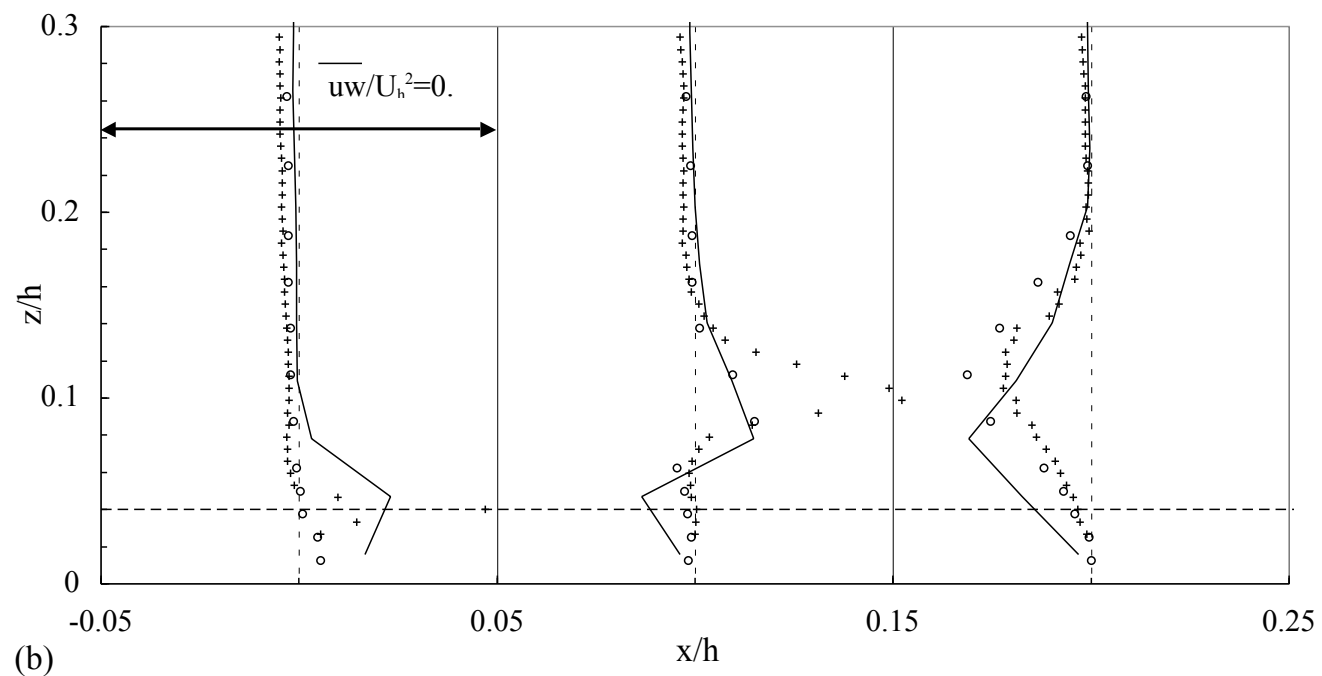

Fig. 15. Shear stress profiles $\left(\overline{u w} / U_{h}^{2}\right)$ over the cube. Symbols as in Figure 11.

\subsubsection{Mean near-surface conditions}

Given that the mean velocity field is not significantly different between the experiment and the calculation one would expect the mean surface pressure field to be similar also. Figure 16 compares the measured and calculated pressure distributions along the axial and transverse centerlines on the top surface, with pressures shown as coefficients as usual (i.e. $\left.C_{p}=\left(p_{s}-p_{o}\right) / \frac{1}{2} \rho U_{h}^{2}\right)$. In these and later figures $x^{\prime}$ is defined as the distance along the spanwise centre-line of the cube, starting from the bottom of the front face. (So $x^{\prime} / h=1$ and $x^{\prime} / h=3$ refer to the top leading edge and the bottom of the rear face, respectively.) The profiles in Fig.16(a) have the expected shape, in that the largest 

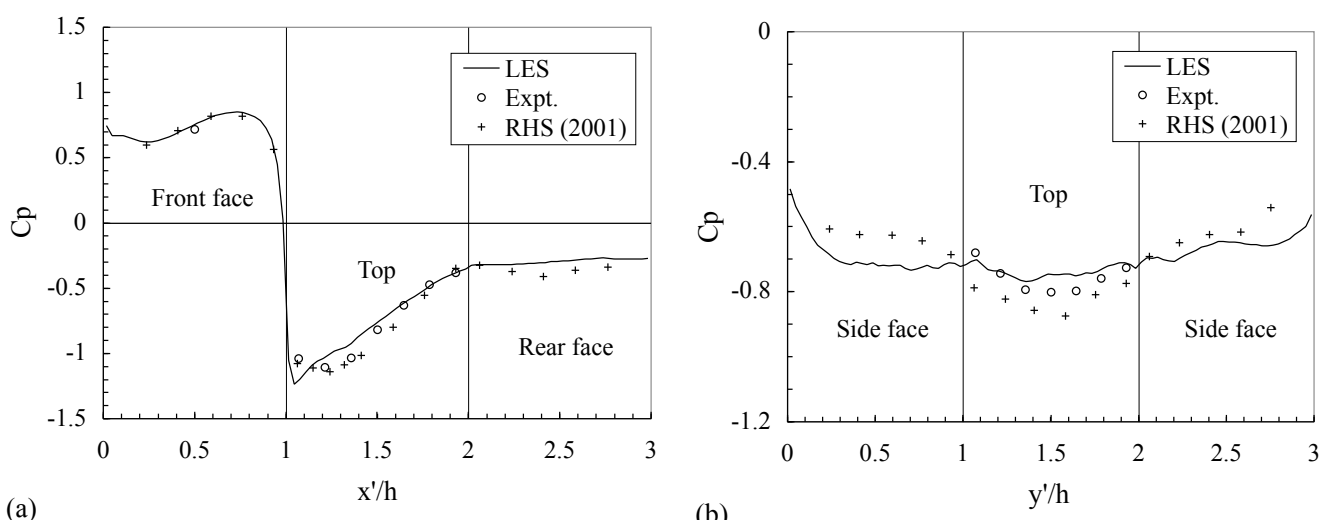

(b)

Fig. 16. Surface pressure distributions on the top surface of the cube. (a): along $y=0$; (b): along $x / h=0.5$ - i.e. the transverse centreline. In (a) the ordinate shows the normalised distance from the front, bottom of the cube up to the top leading edge and thence to the rear leading edge and the bottom of the rear face. In (b) $y^{\prime}=0$ is the bottom central point on a side face. RHS (2001) refers to Richards et al. (2001).

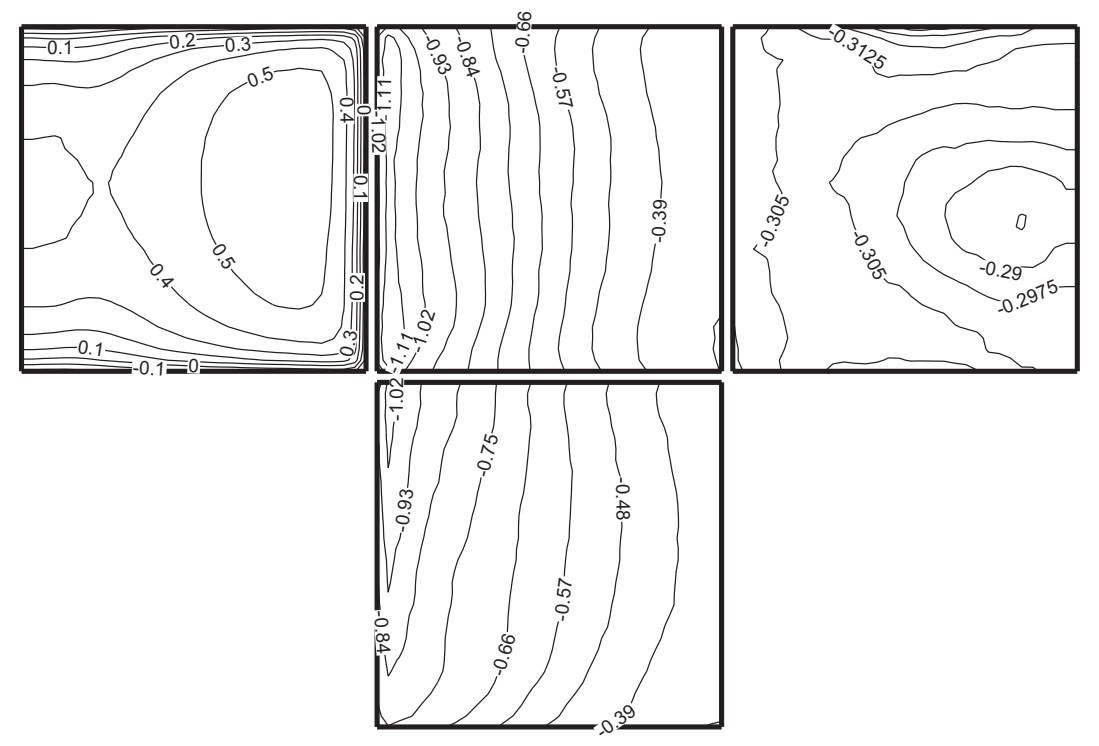

Fig. 17. Mean Cp contours on cube faces. Flow is from left to right with the left and right panels showing the front and back faces, respectively, and the bottom panel showing a side face.

negative pressures occur just beyond separation and are followed by a substantial pressure recovery associated with the attachment process on the top surface, as shown frequently in previous studies (e.g. Castro \& Robins, 1977). Note that the data are similar to the field data (at $R e_{h}=2.8 \times 10^{6}$ ) of Richards et al. (2001) which were obtained in similar conditions (e.g. Jenson number, turbulent intensities, spectra) as those of the current wind-tunnel boundary layer, although of course at a very much higher Reynolds number. Lim et al. (2007) showed that these $C_{p}$ profiles do not depend significantly on 
the Reynolds number. It is particularly encouraging that the LES computations capture the peak negative pressure just beyond the separation at the leading edge. The mesh resolution is thus in that respect quite sufficient. Contours of the mean surface pressure coefficient on all cube faces, derived from the LES computations, are shown in Figure 17. There is a hint of asymmetry on the rear face but, as can be seen from the contour levels shown, the pressure is fairly uniform over this face; no doubt longer averaging times would have removed these slight asymmetries.

Figure 18 shows axial profiles of mean axial velocity just above the top surface of the cube, obtained using PIV at two resolutions, LDA and LES. Field data from sonic anemometers, obtained by Lim et al. (2007), are also included. (Hot wire anemometry cannot be used in this highly turbulent region, of course.) Note first that all data clearly indicate that mean attachment occurs around $x^{\prime} / h=1.75$ - i.e. three-quarters of the distance along the top surface or actually just beyond that point, recognising that the profile was obtained at a distance $0.01 \mathrm{~h}$ above the surface. This is consistent with the implication of the vertical profiles shown earlier (fig.11). Upstream of that location the mean flow is reversed. The data obtained by LES, PIV and (in the field) the sonic anemometers indicate noticeably lower magnitudes of $U / U_{h}$ than given by the LDA. As indicated earlier, this is almost certainly a spatial resolution issue (see Lim et al. 2007) and is the major reason we include these PIV data for comparison with the LES. The grid size of the calculation and the acoustic path length of the sonic anemometers were equivalent to about $h / 32=0.03 h$ and $0.02 h$ respectively, so these techniques would be expected to overestimate $U / U_{h}$ in this region of high shear. Similarly, a $16 \times 16$ pixel interrogation area in analysing the PIV images implies a $1.25 \mathrm{~mm} \times 1.25 \mathrm{~mm}$ area - i.e. $0.016 h \times 0.016 h$, with equivalent doubling and halving of this for $32 \times 32$ or $8 \times 8$ pixel domains. It is clear that reducing the PIV integration area size led to values of $U / U_{h}$ noticeably lower (in magnitude), tending towards the LDA data, which were essentially genuine point measurements. The $16 \times 16$ data are quite close to those obtained using LES (and with the sonic anemometers), so resolution effects are clearly similar in these cases, reflecting the similarity in the normalized sampling volumes.

\subsubsection{Fluctuating near-surface conditions}

The fact that the overall mean flow, as characterized by surface pressures and nearsurface velocities, is not significantly different between the experiment and calculation does not necessarily imply that the same level of agreement would hold for fluctuating quantities like the r.m.s. pressure or the mean square of the velocity fluctuations. Figure 19(a) compares the measured and calculated fluctuating surface pressure profiles along the centreline of the cube. The LES results do in fact show reasonable agreement with the wind tunnel experiments except, not surprisingly perhaps, near the separation region at the leading edge, where the LES velocity data were previously noted as being affected by inadequate resolution (figs. 13 \& 14). However, the LES values are here rather larger than the experimental data, which seems opposite to what one would expect from inadequate spatial resolution. The overall agreement between the fluctuating $C_{p}$ values along the centreline of the cube surface is encouraging, but it seems inconsistent with the variation expected from an increased diffusion implied by the subgrid model, i.e. a lower 'effective' Reynolds number. It should be emphasised that although the mean pressures are largely independent of Reynolds number, the same is not true for fluctuating pressure, as shown 


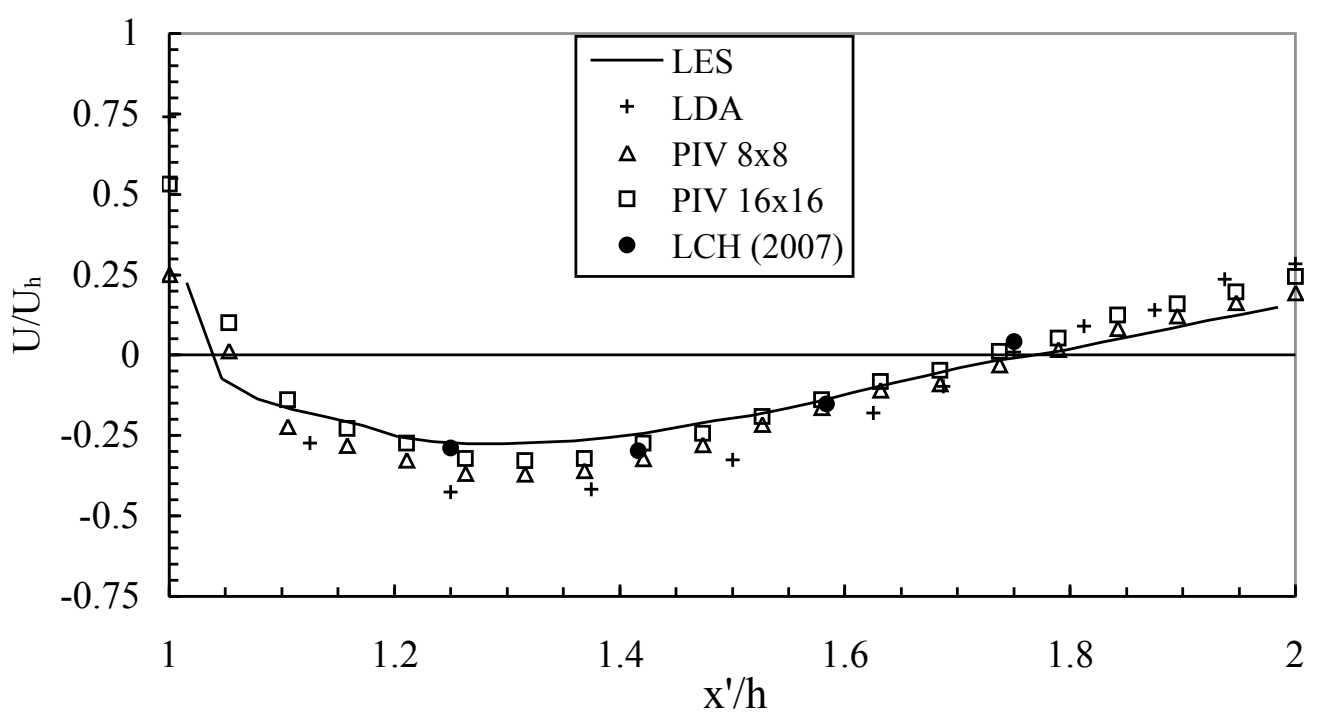

Fig. 18. Streamwise component velocity close to the cube top; $z / h \approx 1.01$ (1.016 for LES).
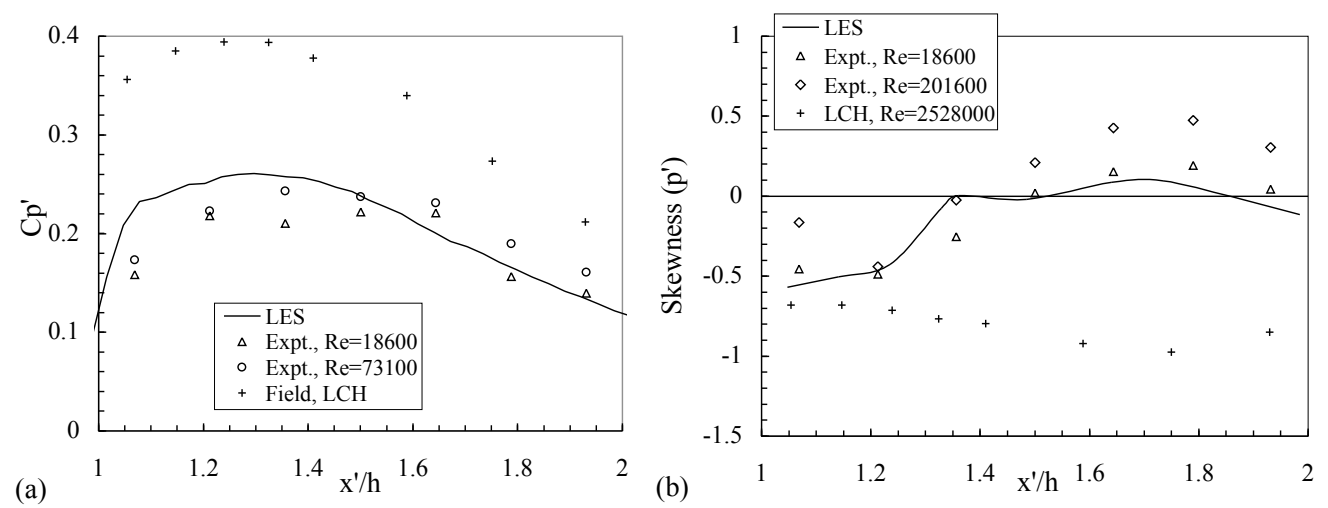

Fig. 19. Fluctuating surface pressure along the axial centreline of the cube. (a): rms $C_{p}$; (b): skewness of $p^{\prime}$.

by Lim et al. (2007); this is emphasised by the inclusion of field data obtained by the latter authors. It would be instructive to run an LES at a much higher Reynolds number, but this has not yet been done (it would require a much finer grid, or a more sophisticated sub-grid model).

This indirect evidence suggests that at least for Reynolds number of $\mathrm{O}(20000)$ the resolution is sufficient to allow capture of a good part of the inertial range in most regions of the flow (see, e.g., fig.4) and this is sufficient to capture fluctuating pressures adequately, so that details of the sub-grid model are not too significant. This is likely always to be true for flows, like the present one, which are heavily dominated by the dynamics of the large scale structures - in this case, those generated by the body itself. Direct evidence for this conclusion is provided by spectra. It was noted earlier (fig.4) 

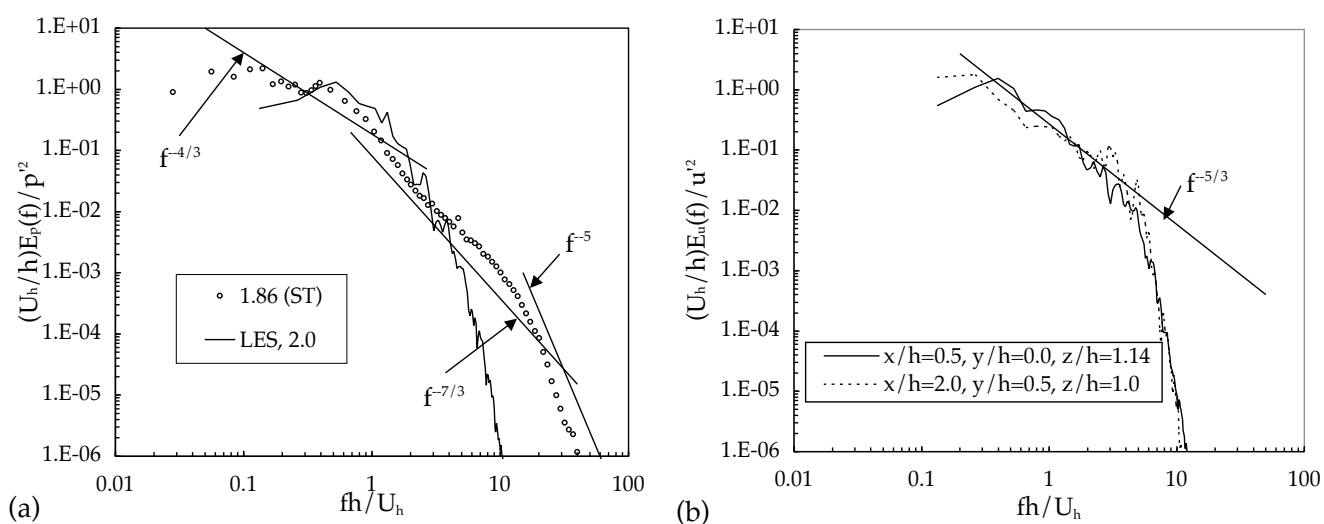

Fig. 20. (a): surface pressure spectra at the centre of the top surface of the cube; numbers in the legend refer to $R e_{h} \times 10^{-4}$. (b) LES axial velocity spectra just above the centre of the top surface $(z / h=1.14)$ and at a point in the wake.

that in the upstream flow, the LES spectra are in good agreement with experiment over a satisfyingly large inertial region. The same is true for spectra obtained around the cube, as demonstrated by Figure 20. This shows LES surface pressure spectra obtained at the centre of the top surface (fig.20a), compared with measured data at about the same Reynolds number. The latter are fully discussed by Lim et al. (2007) and clearly show the expected $-\frac{7}{3}$ inertial region over at least a decade. Fig.20b shows LES axial velocity spectra at two locations - one just above the centre of the cube and another in the downstream wake region. Although the LES surface pressure spectra perhaps only marginally show an inertial region, both velocity spectra show at least a decade of an inertial $-\frac{5}{3}$ region. In all cases (and in fig.4), the premature roll-off - starting around $f h / U_{h} \approx 4$ - is fixed by the mesh size; finer meshes would allow continuation of the inertial range. We emphasise too that the domain length fixes the lowest possible $f h / U_{h}$ value and the finite averaging time (much lower than in experiments of course) causes the obvious scatter in the spectral data.

Figure 19(b) shows the skewness of the fluctuating pressure along the top surface centreline. Again, there is quite good agreement between the LES calculation and the laboratory experiment. Note again the very different field values, which suggest stronger and/or more frequent negative peaks in pressure, which is consistent too with the higher rms values seen in Fig.19(a). Since the skewness (unlike the mean square) is a measure of the relative importance of the extreme positive and negative fluctuations, this agreement suggests that extreme pressures are quite well captured by the LES. This is emphasised in Figure 21, which shows the probability density function of the fluctuating surface pressure obtained at a point on the top centreline of the cube at a distance $0.06 \mathrm{~h}$ from the leading edge. Within the uncertainty of the LES data, arising from the relatively short sampling duration (compared with that for the microphone), there is reasonable overall agreement between LES and the experiment. The values of the probability density function, $p(p)$, in the extreme low pressure region (large negative values of $p / \sigma_{p}$ ) are significantly higher than would be expected if the pressure fluctuations were Gaussian, but it should be noted that the LES data fall off rather more rapidly than suggested by the experiments. This is almost certainly a result of the limited spatial resolution. One would expect better 


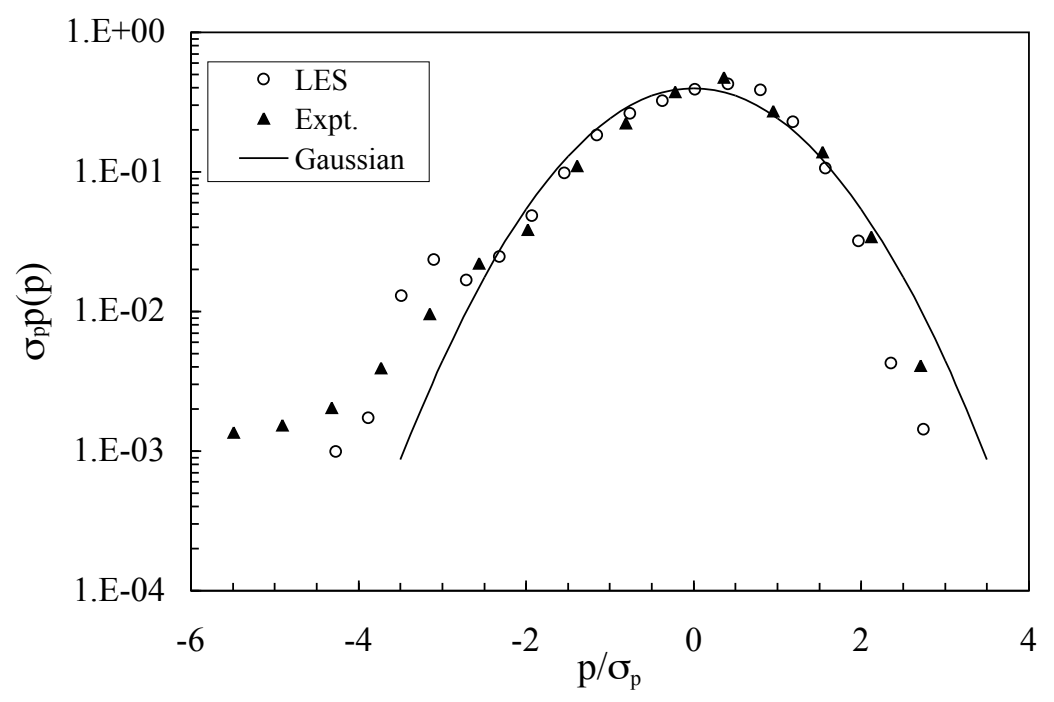

Fig. 21. Normalised probability density of the fluctuating pressure at $x^{\prime} / h=1.06, z / h=1.0$.

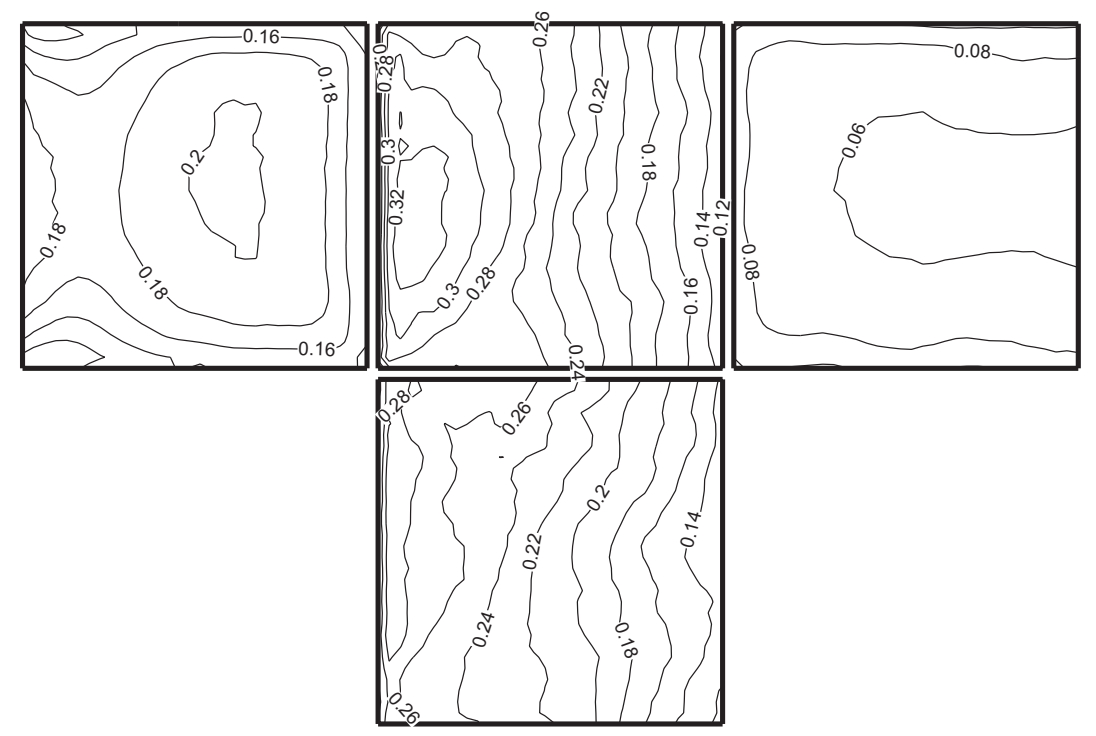

Fig. 22. Cp' contours on cube faces.

agreement for finer grids - as indeed is suggested by Nozawa \& Tamura's (2002) findings - although LES will always be limited to some extent, in this respect. It might be possible to parameterise the negative tail of the pdf (characterising the extreme values), based on experimental data like those here or using extreme value theory (e.g. Smith, 1989, Xie et al. , 2007), but we have not attempted this.

For completeness, we show in Figure 22 the contours of fluctuating (rms) pressure coefficient, $C_{p}^{\prime}$, on the faces of the cube - corresponding to the mean pressure contours 


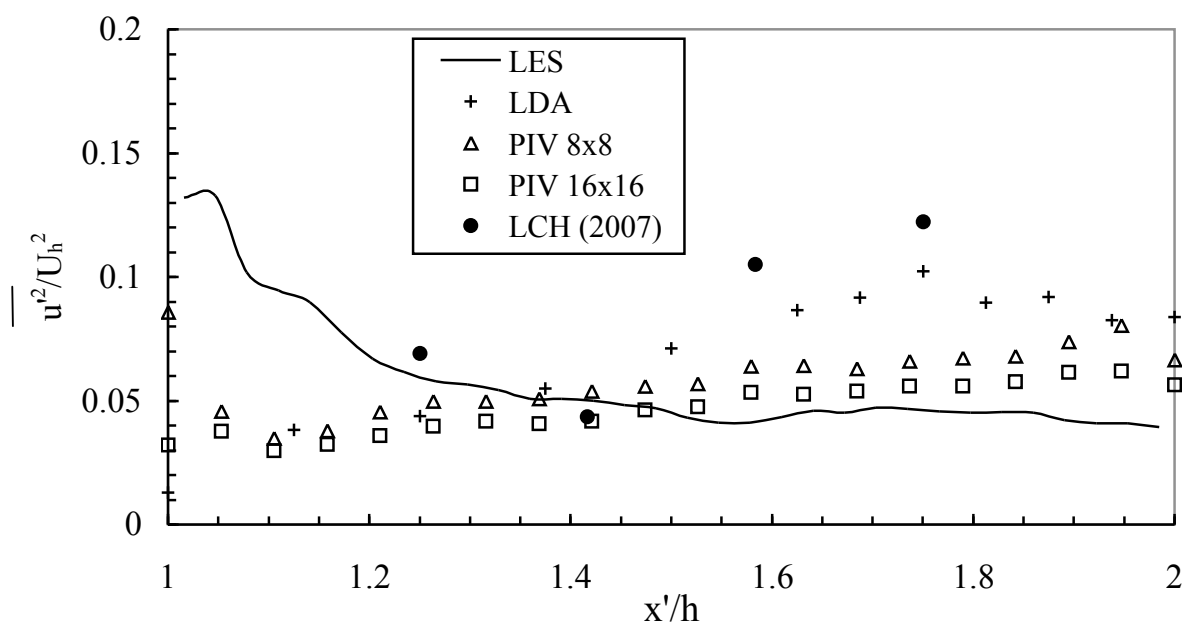

Fig. 23. Streamwise component axial stress profiles on the cube top.

shown in fig.17. The contours are less smooth than the mean pressure contours and, again, there is some sign of slight asymmetry; longer averaging times would undoubtedly remove these features.

Although the calculated fluctuating pressures are similar to the measured values, there are noticeable differences in the fluctuating velocities just above the cube surface, as is clear in Figure 23, which shows the axial turbulence stress along the top-surface centreline (corresponding to the mean flow data in fig.18). The axial turbulence levels are significantly over-predicted near the leading edge, but under-predicted over the rear half of the cube. In the latter region, the LES data are close to the less-well resolved PIV data, as expected. Likewise, near the leading edge, the strong shear coupled with widely varying instantaneous flow directions require finer resolution (as noted in the context of fig. 13). Since it is difficult to see how under-resolution would lead to an over-prediction of total turbulence energy, unless locally the sub-grid model fails to dissipate enough energy, we conclude that the other two components must be under-predicted near the leading edge and the differences arise because of the non-axial mean flow direction (so that individual normal stresses cannot be interpreted in the usual way, as noted earlier).

\section{Conclusions}

A large eddy simulation of a turbulent flow past a cubic obstacle has been carried out alongside an experimental study of the same flow. The results presented include a detailed comparison between measurements and LES computations of both the inflow boundary layer and the flow field around a cube including the near wake. The computational mesh was uniform, as this aided rapid convergence of the multigrid solver, and had a grid spacing of $h / 32$. The results suggest that this is adequate to capture the major features of the flow. Mean and fluctuating surface pressures appear to be well-captured although there are a few regions (principally where the velocity shears are very high) in which the spatial resolution is perhaps not sufficient. However, this does not seem to lead to a failure to 
capture the appropriate large-scale dynamics of the flow. Further study, concentrating on the issue of sub-grid scale adequacy in the regions of relatively steady and strongly concentrated vorticity that occur in the case of a cube at $45^{\circ}$ is forthcoming. Meanwhile, the present work confirms the conclusion of corresponding studies undertaken in channel flows: LES is now a viable tool for use in wind engineering problems concerning flow over isolated bodies, despite the necessity (and inconvenience) of generating an appropriate upstream boundary layer.

We are grateful to the UK's Engineering \& Physical Sciences Research Council for support (of HCL) under grant no. GR/S12135/01 and to the generous help from technical staff at the Southampton University. We also wish to thank EnFlo's technical support staff, principally Dr Paul Hayden and Mr Tom Lawton, for their continued support of the instrumentation software used in the experiments described in this paper.

\section{References}

Castro, I.P. and Graham J.G.R., 1999. Computational Wind Engineering: The Way Ahead? Proc. Instn. Civ. Engrs. Structs \& Bldgs. 134, 275-277.

Castro, I.P. and Robins, A.J., 1977. The flow around a surface mounted cube in uniform and turbulent streams. J. Fluid Mech. 79, 307-335.

Cook, N.J., 1978. Wind tunnel simulation of the adiabatic atmospheric boundary layer by roughness. J. Wind Eng. Ind. Aerodyn. 3, 157-176.

ESDU, 1985. Characteristics of atmospheric turbulence near the ground. Part II: single point data for strong winds (neutral atmosphere). Engineering Sciences Data Unit, Item 85020.

Ferziger, J.H., 1990. Approaches to turbulent flor computation: application to flow over obstacles. J. Wind Eng. Ind. Aero. 35, 1-19.

Ghosal, S., Luna, T.S., Moin, P. and Akselvoll, K., 1995. A dynamic localization model for large eddy simulation of turbulent flows. J. Fluid Mech. 286, 229-255.

Hart, D.P., 1999. Super-resolution PIV by recursive local correlation. J. Visualization, 10.

Klein, M., Sadiki, A. and Janicka, J., 2003. A digital filter based generation of inflow data for spatially developing direct numerical simulation or large eddy simulation. $J$. Comp. Phys. 186, 652-665.

Krajnovic, S., Muller, D. and Davidson, L., 1999. Comparison of two one-equation subgrid models in recirculating flows. In Direct and Large-Edyy Simulation III, P. Voke, N.D. Sandham, L. Kleiser (eds.), Kluwer, 63-74.

Lesieur, M. and Metais, O., 1996 New trends in large-eddy simulation of turbulence. Ann. Rev. Fluid. Mech. 28, 45-82.

Lim, H.C., Castro, I.P. and Hoxey, R.P., 2004. Flow over a cube in a thick boundary layer - revisited. In Proc.6th UK Conf. On Wind Engineering, September 2004. Cranfield University, UK.

Lim H.C. and Thomas T.G., 2005. LES of Flow around a Cubic Obstacle placed in a Turbulent Boundary Layer. In The 2nd Symposium on Advanced Turbulence Modelling for Industrial CFD, October, 2005, Esprito Santo, Brazil.

Lim, H.C., Castro, I.P. and Hoxey, R.P., 2007. Bluff bodies in deep turbulent boundary 
layers: Reynolds-number issues . J. Fluid Mech. 571, 97-118.

Mason, P. J., 1994. Large-eddy simulation: a critical review of the technique. Q.J.R. Meteorol. Soc. 28, 1-26.

Mason, P.J. and Thomson, D.J., 1992. Stochastic backscatter in large-eddy simulations of boundary. J. Fluid Mech. 242, 51-78.

Melbourne, W.H., 1980 Turbulence effects on maximum surface pressures: a mechanism and possibility of reduction. In Wind Engineering (ed. J.E.Cermak). 541-551, Pergamon.

Murakami, S., Mochida, A. and Hibi, K. 1987. Three-dimensional numerical simulation of air flow around a cubic model by means of Large-Eddy Simulation. J. Wind Eng. Ind. Aerodyn. 25, 291-305.

Murakami, S., 1997. Current status and future trends in computational wind engineering. J. Wind Eng. Ind. Aerodyn. 67\&68, 3-34.

Nozawa, K. and Tamura, T., 2002. Large eddy simulation of the flow around a lowrise building immersed in a rough-wall turbulent boundary layer. J. Wind Eng. Ind. Aerodyn. 90, 1151-1162.

Ono, Y., Tamura, T. and Kataoka, H., 2006. LES analysis of unsteady characteristics of conical vortex on a flat roof. Paper in 4th International Symposium on Computational Wind Engineering (CWE2006, pp.373-376, Yokohama.

Perry, A.E., Lim, K.L. and Henbest, S.M., 1987. An experimental study of the turbulence structure in smooth and rough wall turbulent boundary layers. J. Fluid Mech. 177, 437-466.

Rai, M.M and Moin, P., 1993. Direct numerical simulation of transition and turbulence in a spatially evolving boundary layer. J. Comput. Phys. 109, 169-192.

Richards, G.M., Hoxey,R.P. and Short, L.J., 2001. Wind pressures on a $6 \mathrm{~m}$ cube . J. Wind Eng. Ind. Aero. 89, 1553-1564.

Richards, G.M. and Hoxey, R.P., 2002. Unsteady flow on the sides of a $6 \mathrm{~m}$ cube . J. Wind Eng. Ind. Aero. 90, 1855-1866.

Rodi, W., 2002. Large-eddy simulation of the flow past bluff bodies. In Closure strategies for turbulent and transitional flows, B.E. Launder, N.D. Sandham (eds.), CUP, 361391.

Schumann, U., 1975. Subgrid-scale model for finite difference simulations of turbulent in plane channels. J. Comput. Phys. 18, 376-404.

Shah, K.B. 1998. Large-eddy simulations of flow past a cubic obstacle. PhD Thesis, Dept. of Mech. Eng., Stanford University.

Shah, K.B. and Ferziger, J.H., 1996. Large-eddy simulations of flow past a cubical obstacle. Thermosciences Div. Rep. TF-70., Dept. of Mech. Eng., Stanford University.

Shah, K.B. and Ferziger, J.H., 1997. A fluid mechanician's view of wind engineering: Large-eddy simulation of flow past a cubical obstacle. J. Wind Eng. Ind. Aero. 67\&68, 211-224.

Shavit, U.a., Lowe, R.J. and Steinbuck, J.V., 2007. Intensity Capping: a simple method to improve cross-correlation PIV results . Exp. in Fluids 42(2), 225-240.

Smagorinsky, J., 1963. General circulation experiments with primative equations: I. The basic experiment. Mon. Weather Rev. 91, 99-164.

Smith, R.L., 1989. Extreme value analysis of environmental time series: an application to trend detection in ground level ozone (with discussion). Statistical Science 4, 367-393.

Thomas, T.G. and Williams, J.J.R., 1997. Development of a parallel code to simulate 
skewed flow over a bluff body. J. Wind Eng. Ind. Aerodyn. 67\&68, 155-167.

Thomas, T.G. and Williams, J.J.R., 1999 Generating a wind environment for large hedi simulation of bluff body flows. J. Wind Eng. Ind. Aerodyn. 82, 189-208.

Wernet, M.P., 2005. Symmetric phase only filtering: a new paradigm for DPIV data processing. Measurement Sci. \& Tech. 16(3), 601-618.

Xie, Z.T., Voke, P.R., Hayden, P. and Robins, A.G., 2004. Large-eddy simulation of turbulent flow over a rough surface. Boundary-Layer Met. 111, 417-440.

Xie, Z.T. and Castro, I.P., 2008. Efficient generation of inflow conditions for Large-Eddy Simulations of street-scale flows. J. Flow, Turb. 65 Comb. 81, 449-470.

Xie, Z.T., Hayden, P., Robins, A.G. and Voke, P.R., 2007. Modelling extreme concentration from a source in a turbulent flow over rough wall. Atmos. Environ. 41, 3395-3406.

Yakhot, A., Anor, T., Heping, L. and Nikitin, N., 2006. Direct numerical simulation of turbulent flow around a wall-mounted cube: spatio-temporal evolution of large-scale vortices. J. Fluid Mech. 566, 1-9. 